\title{
Scalable Evaluation of Trajectory Queries over Imprecise Location Data
}

\author{
Xike Xie, Member, IEEE, Man L. Yiu, Reynold Cheng, Member, IEEE, and Hua Lu Member, IEEE
}

\begin{abstract}
Trajectory queries, which retrieve nearby objects for every point of a given route, can be used to identify alerts of potential threats along a vessel route, or monitor the adjacent rescuers to a travel path. However, the locations of these objects (e.g., threats, succours) may not be precisely obtained due to hardware limitations of measuring devices, as well as complex natures of the surroundings. For such data, we consider a common model, where the possible locations of an object are bounded by a closed region, called "imprecise region". Ignoring or coarsely wrapping imprecision can render low query qualities, and cause undesirable consequences such as missing alerts of threats and poor response rescue time. Also, the query is quite time-consuming, since all points on the trajectory are considered. In this paper, we study how to efficiently evaluate trajectory queries over imprecise objects, by proposing a novel concept, $u$-bisector, which is an extension of bisector specified for imprecise data. Based on the $u$-bisector, we provide an efficient and versatile solution which supports different shapes of commonly-used imprecise regions (e.g., rectangles, circles, and line segments). Extensive experiments on real datasets show that our proposal achieves better efficiency, quality, and scalability than its competitors.
\end{abstract}

Index Terms-Trajectory query, possible nearest neighbor, imprecise object, $u$-bisector

\section{INTRODUCTION}

$\mathrm{T}$ RAJECTORY queries retrieve nearby objects for a given route. Such queries are useful in various domains including transportation and facility management. For example, in the air and shipping industries where safety is the top priority, it is very important to identify potential threats along the route of a flight or a vessel and give alerts in advance. Such threats are exemplified by volcanic ashes for flights in North Europe [1] and icebergs for vessels in U.S. [2]. Due to the limited capacity for a radar system in tracking multiple targets [3], it would be beneficial to focus on those closest threats. As another example, trajectories can also represent the pipelines for transporting oil, gas, water, etc. When a section of a pipeline is broken, it causes economic loss and potential hazard. The authority therefore needs to call up the technicians nearest to the damage spot in order to fix the problem [4] as soon as possible.

One fundamental challenge in such scenarios is that the measured locations of objects (e.g., clouds of volcanic ash, icebergs, or people) are imprecise. Such imprecise locations result from: (i) limited resolution of the measure device, (ii) infrequent measurement, and/or (iii) environmental factors.

- X. Xie and H. Lu are with the Department of Computer Science, Aalborg University, Aalborg DK-9220, Denmark.

E-mail: \{xkxie,luhua\}@cs.aau.dk.

- M. L. Yiu is with the Department of Computing, Hong Kong Polytechnic University, Hong Kong. E-mail: csmlyiu@comp.polyu.edu.hk.

- $R$. Cheng is with the Department of Computer Science, University of Hong Kong, Hong Kong. E-mail: ckcheng@cs.hku.hk.

Manuscript received 10 Oct. 2012; revised 29 Apr. 2013; accepted 30 Apr. 2013. Date of publication 12 May 2013; date of current version 10 July 2014.

Recommended for acceptance by C. Shahabi.

For information on obtaining reprints of this article, please send e-mail to: reprints@ieee.org, and reference the Digital Object Identifier below.

Digital Object Identifier 10.1109/TKDE.2013.77
In the transportation example, the threats (icebergs or volcanic ashes) are often detected by remote sensing technologies like satellite imaging. Such technologies usually work at low sensing frequency because of cost constraints, and thus render the measured locations stale for objects. Furthermore, icebergs (volcanic ashes) can move depending on the ocean current (wind) speed. In the pipeline example, a technician may have a GPS device for location tracking [4], where GPS reports locations with measurement errors subject to terrain and climate conditions [5].

Consequently, trajectory queries have to handle such imprecise objects whose locations cannot be precisely determined. Table 1 summarizes these aforementioned two kinds of applications that involve imprecise objects.

A common way to model an imprecise object is to use so-called imprecise region [8]-[14], which is a closed region covering all possible position during a time interval. Fig. 1 illustrates imprecise regions of different shapes that are seen in GPS, RFID, and road network applications.

In this paper, we study the problem of searching imprecise objects close to a given query trajectory. Fig. 2(a) shows a query trajectory $T=\left\{q_{1}, q_{2}, q_{3}, q_{4}\right\}$ and a set of imprecise objects $\mathrm{O}_{1}, \mathrm{O}_{2}, \mathrm{O}_{3}$. The query result (Fig. 2(b)) is represented in a compact way by partitioning the query trajectory into segments such that all locations within the same segment share the same result. In this example, $\mathrm{O}_{2}$ is the definite nearest neighbor to segment $\left[s_{2}, s_{3}\right]$. On the other hand, $O_{1}$ and $O_{2}$ are possible nearest neighbors (PNNs) to segment $\left[s_{1}, s_{2}\right]$ because both of them have potential to be the closest object for any location between $s_{1}$ and $s_{2}$.

Determining the query results over imprecise objects is technically challenging, as the geometries of the imprecise regions must be considered. A simple solution is to replace the imprecise region of each object with a central point 
TABLE 1

Summary of Applications

\begin{tabular}{|c||c|c|}
\hline Application & Route Safety & Pipeline Maintenance \\
\hline Trajectory & route of a flight or vessel & fuel or water pipeline \\
\hline Objects & volcanic ashes or icebergs & technicians \\
\hline Localization & remote sensing & GPS \\
\hline $\begin{array}{c}\text { Imprecise } \\
\text { data source }\end{array}$ & $\begin{array}{l}\text { resolution, environment, } \\
\text { infrequent measurement }\end{array}$ & $\begin{array}{c}\text { GPS error, } \\
\text { terrain, climate }\end{array}$ \\
\hline
\end{tabular}

(shown as a grey dot in Fig. 2(c)). Accordingly, the single closest object is associated with the corresponding segment in the query result, as shown in Fig. 2(d). For instance, the closest object to location $q_{2}$ appears to be object $O_{1}$ only and object $\mathrm{O}_{2}$ is missing from the result. Recall that object $\mathrm{O}_{2}$ also has the possibility to be a closest object to location $q_{2}$ as shown in Fig. 2(a) and (b).

In the aforementioned application scenarios, the "center simplification" approach causes undesirable consequences such as missing threat alerts and poor response time. In the flight/vessel example, modeling threats as imprecise regions prioritizes the safety in all cases, whereas the ignorance of imprecise regions can cause potential dangers. In the pipeline example, a technician seemingly close to (far from) the broken pipeline section may be actually far from (close to) it due to the location imprecision. Calling up such a technician would incur longer time to respond to the emergency. It is important to call up all technicians likely to be close to the damage spot, in order to fix the problem as soon as possible.

An alternative to simplify the trajectory query is the "sampling approach", which considers only those positions at every fixed length on the query trajectory and computes the nearby objects for each such sample. However, deciding the sampling rate is a dilemma in this approach. A high sampling rate incurs huge computation costs, while a low rate can miss many answers. Referring to Fig. 2(a), the query result changes only at a few positions $\left(s_{1}, s_{2}, s_{3}, s_{4}\right)$. It is not clear how to decide the correct sampling rate in order to get these answers.

Neither the center simplification approach nor the sampling approach solves the trajectory queries over imprecise objects. In fact, our preliminary experiments show that they cannot guarantee correct and complete query results. Therefore, we develop a solution that can accurately compute a trajectory query on imprecise objects in this paper. A special case of our problem, finding the closest precise

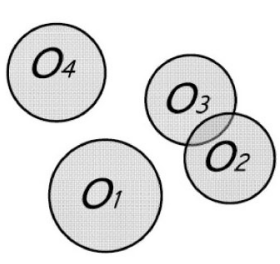

(a)

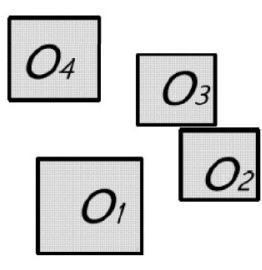

(b)

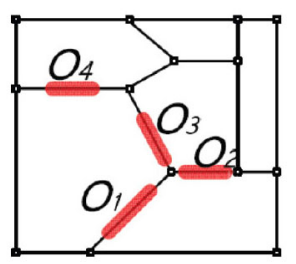

(c)
Fig. 1. Imprecise regions. (a) Circle can be used to describe the position uncertainty of a person or vehicle tracked by GPS [6]. (b) Rectangle can be a person's imprecise region when the RFID-based indoor tracking works on the room level [7]. (c) Line segment is used, when a vehicle is moving in a road network [6].

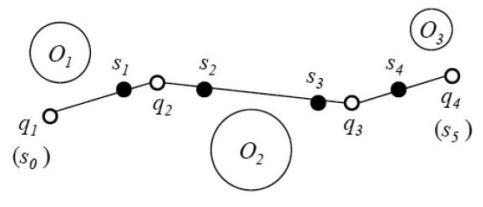

(a)

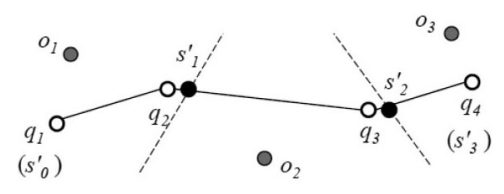

(c)

\begin{tabular}{|c|c|}
\hline Segment & Result \\
\hline \hline$\left[s_{0}, s_{1}\right]$ & $O_{1}$ \\
\hline$\left[s_{1}, s_{2}\right]$ & $O_{1}, O_{2}$ \\
\hline$\left[s_{2}, s_{3}\right]$ & $O_{2}$ \\
\hline$\left[s_{3}, s_{4}\right]$ & $O_{2}, O_{3}$ \\
\hline$\left[s_{4}, s_{5}\right]$ & $O_{3}$ \\
\hline
\end{tabular}

(b)

\begin{tabular}{|c|c|}
\hline Segment & Result \\
\hline \hline$\left[s_{0}^{\prime}, s_{1}^{\prime}\right]$ & $O_{1}$ \\
\hline$\left[s_{1}^{\prime}, s_{2}^{\prime}\right]$ & $O_{2}$ \\
\hline$\left[s_{2}^{\prime}, s_{3}^{\prime}\right]$ & $O_{3}$ \\
\hline
\end{tabular}

(d)
Fig. 2. Example trajectory query. (a) Imprecise objects. (b) Result. (c) Precise objects. (d) Result.

points for a given query trajectory, was studied by Tao et al. [15]. The authors used the (perpendicular) bisectors of each pair of consecutive points to derive the query answer. For example, in Fig. 2(c), the point $s_{1}^{\prime}$ is the intersection between the query trajectory and the bisector (in dashed lines) of precise points $\mathrm{O}_{1}$ and $\mathrm{O}_{2}$. Likewise, $s_{2}^{\prime}$ is derived by the bisector of $\mathrm{O}_{2}$ and $\mathrm{O}_{3}$.

We extend the bisector concept to $u$-bisector in order to support imprecise objects. Fig. 3 illustrates the $u$-bisectors for circular and rectangular imprecise regions. Note that a $u$-bisector is not a straight line anymore for two objects $o_{i}$ and $o_{j}$. Instead, it becomes a pair of curves, namely $b_{i}(j)$ and $b_{j}(i)$, that partition the domain space into three parts: (1) the left part, where points are absolutely closer to $O_{i}$ than to $O_{j}$; (2) the right part, where points are absolutely closer to $O_{j}$ than to $O_{i}$; and (3) the middle part, where points can be closer to either $O_{i}$ or $O_{j}$. We call the region enclosed by a $u$-bisector half as a half-space. For example, in Fig. 3(a), the left of $b_{i}(j)$ is a half-space, and so is the right of $b_{j}(i)$. We make use of half-spaces and $u$-bisectors to answer a trajectory query.

In practice, it is challenging to compute the intersections between the query trajectory and $u$-bisectors. As shown in Fig. 3, u-bisectors can be hyperbolic curves (Fig. 3(a)), or polylines (Fig. 3(b)). Furthermore, these $u$-bisectors may intersect the query trajectory at multiple points. Our solution avoids generating $u$-bisectors for all pairs of imprecise objects by employing a filter-refinement framework. In the filtering phase, candidate objects that may be the closest to each query segment are obtained. In the refinement phase,

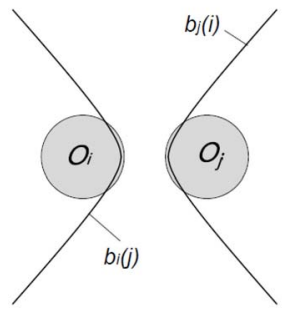

(a)

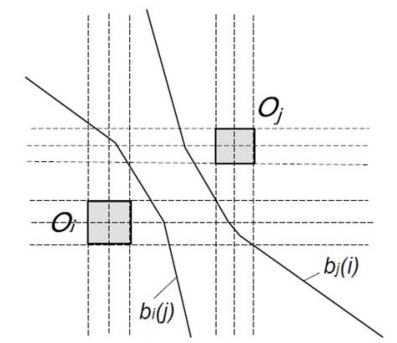

(b)
Fig. 3. u-bisector for imprecise regions. (a) Circular imprecise region. (b) Rectangle imprecise region. 
TABLE 2

Notations and Meanings

\begin{tabular}{|c|c|}
\hline Notation & Meaning \\
\hline$D$ & Domain space (a square) \\
\hline$|\cdot|$ & the area of a region \\
\hline$O$ & a set of imprecise objects $\left(O_{1}, O_{2}, \ldots, O_{n}\right)$ \\
\hline$M B C\left(O_{i}\right)$ & minimum bounding circle of object $O_{i}$ \\
\hline$\odot_{i}\left(c_{i}, r_{i}\right)$ & circle $\odot_{i}$ with center $c_{i}$ and radius $r_{i}$ \\
\hline$\odot\left(p, O_{i}\right)$ & circle centered $p$ and internally tangent with $O_{i}$ \\
\hline$\overline{s e} /[s, e]$ & line segment with two end points $s$ and $e$ \\
\hline$b_{i}(j)$ & $O_{i}$ and $O_{j}$ 's $u$-bisector half, which is closer to $O_{i}$ \\
\hline$H_{i}(j)$ & half space cut by $b_{i}(j)$, which is closer to $O_{i}$ \\
\hline$s_{i \vdash j}$ & intersection between a line segment and $b_{i}(j)$ \\
\hline$s_{i \dashv j}$ & $\begin{array}{l}\text { intersection between a line segment and } b_{j}(i) \text {, } \\
\text { which is equivalent to } s_{j \vdash i}\end{array}$ \\
\hline$q$ & a query point \\
\hline$\oplus$ & Minkowski sum \\
\hline $\mathcal{T} /|\mathcal{T}|$ & trajectory $\mathcal{T} /$ length of trajectory $\mathcal{T}$ \\
\hline $\mathbb{T}(\mathcal{T})$ & trajectory tree constructed for trajectory $\mathcal{T}$ \\
\hline$\Psi(\mathcal{L})$ & ternary tree constructed for line-segment $\mathcal{L}$ \\
\hline
\end{tabular}

we develop a novel technique called tenary decomposition to derive the final answers accurately. We show theoretically and experimentally that our solution is efficient and scalable. Moreover, our solution can easily adapt to imprecise objects of arbitrary shapes to other shapes (e.g., circles, rectangles, line segments, etc.) that are required in different applications.

This paper substantially extends our previous work [16] in several aspects. First, we theoretically prove that a halfspace is convex for arbitrary shaped imprecise objects (Section 4.1). Second, we extend the query techniques from supporting circular imprecise objects to objects of arbitrary shapes (Section 4.2). Third, we derive a novel analysis model to estimate the selectivity for trajectory queries (Section 5). Fourth, we conduct extensive additional experiments to evaluate the new proposals (Section 6.3).

The rest of this paper is organized as follows. Section 2 defines the trajectory query we study and presents two query evaluation approaches. Section 3 elaborates on a simplified yet fundamental case where a query trajectory is a single line segment. Section 4 proposes generalized techniques to support different shaped imprecise regions of objects. Section 5 designs an analysis model for trajectory queries. Section 6 presents the experiment results. Section 7 discusses the related works and finally Section 8 concludes the paper. The notations used throughout the paper are listed in Table 2.

\section{Trajectory Possible Nearest NeIGHBor Queries}

\subsection{Problem Definitions}

We first introduce the definition of PNNQ (studied in [6]), which is used to define the query studied in this paper. Let $q$ be a point, and $O_{i}$ an imprecise object from a set $O$. We use $\operatorname{dist}_{\min }\left(q, O_{i}\right)$ and $\operatorname{dist}_{\max }\left(q, O_{i}\right)$ to denote the minimum and maximum distances between $q$ and $O_{i}$, respectively.

Definition 1 (Possible Nearest Neighbor Query (PNNQ)). Given a set of imprecise objects $O$ and a query point $q$, the result of the PNNQ query is a set $P N N Q(q)=\left\{O_{i} \in O\right.$ । $\left.\forall O_{j} \in O\left(\operatorname{dist}_{\max }\left(q, O_{j}\right) \geq \operatorname{dist}_{\min }\left(q, O_{i}\right)\right)\right\}$.
In Fig. 2(a), $P N N Q\left(q_{2}\right)=\left\{O_{1}, O_{2}\right\}$ implies that either $O_{1}$ or $\mathrm{O}_{2}$ could be the $N N$ of the query point $q_{2}$. By extending the concept of $P N N Q$ to all points in a query trajectory $\mathcal{T}$, we define the trajectory possible nearest neighbor query $(T P N N Q)$ which returns $P N N Q$ for all the points in $\mathcal{T}$. In other words, the query returns $\{\langle q, P N N Q(q)\rangle\}_{q \in \mathcal{T}}$. To get a compact representation of the query result, we merge all consecutive trajectory points that have the same $P N N Q$. The formal definition of TPNNQ is given below.

Definition 2 (Trajectory Possible Nearest Neighbor Query

(TPNNQ)). Given a set of imprecise objects $O$ and a query trajectory $\mathcal{T}$, the answer for the TPNNQ query is a set of tuples $R=\left\{\left\langle T_{i}, R_{i}\right\rangle \mid T_{i} \subseteq \mathcal{T}, R_{i} \subseteq O\right\}$, where $\operatorname{PNNQ}(q)=$ $R_{i}\left(\forall q \in T_{i}\right)$, and $T_{i}$ is a continuous segment in $\mathcal{T}$.

In other words, the TPNNQ splits $\mathcal{T}$ into a set of consecutive segments $\left\langle T_{1}, T_{2}, \ldots, T_{t}\right\rangle$ where each $T_{i}$ is a sub-trajectory of $\mathcal{T}$, such that all positions in a given $T_{i}$ have the same possible nearest neighbors. Formally, $\forall q_{i}, q_{j} \in T_{i}, \operatorname{PNNQ}\left(q_{i}\right)=\operatorname{PNNQ}\left(q_{j}\right)$. We call each $T_{i}$ a validity interval. Accordingly, we call the connection point of two consecutive intervals turning point. Such a turning point indicates the change of $P N N Q$ answers. An example for a TPNNQ over three imprecise objects $\left\{\mathrm{O}_{1}, \mathrm{O}_{2}, \mathrm{O}_{3}\right\}$ is shown in Fig. 2(c). The trajectory query $\mathcal{T}\left(s_{0}, s_{5}\right)$ is split into 5 segments. Also, point $s_{1}$ is the turning point for segments $T\left(s_{0}, s_{1}\right)$ and $T\left(s_{1}, s_{2}\right)$. It is apparent that finding turning points is crucial for evaluating TPNNQ. This is however a non-trivial task for imprecise location data. We propose an effective technique for this task in Section 2.2, and develop algorithms on top of it to evaluate TPNNQ in Section 2.3.

There are two major differences between the results on imprecise objects and precise objects. Comparing Fig. 2(c) and (a): (1) the imprecise case could have more result tuples (5 compared to 3); (2) a query point in imprecise case might return a set of PNNs instead of a single object. These observations indicate that the previous techniques for trajectory queries over precise objects [15] do not solve TPNNQ.

\subsection{Finding Turning Points with $u$-bisectors}

Given a set of imprecise objects and a query trajectory, deriving the turning points on the trajectory is the crucial step for answering TPNNQ. To address that, we first investigate the $u$-bisector for imprecise objects. In general, the $u$-bisector splits the domain space into several parts, such that query points on different parts could have different PNNs. After that, the turning points are decided by finding the intersections of the u-bisectors and the query trajectory.

Definition 3. Given two imprecise objects $O_{i}$ and $O_{j}$, their $u$ bisector consists of two curves: $b_{i}(j)$ and $b_{j}(i)$. The $u$-bisector half $b_{i}(j)$ is a set of points satisfying

$$
b_{i}(j)=\left\{z: \operatorname{dist}_{\max }\left(z, O_{i}\right)=\operatorname{dist}_{\min }\left(z, O_{j}\right)\right\}
$$

The curve $b_{i}(j)$ splits the domain space into two parts: $H_{i}(j)$ and $\overline{H_{i}(j)}$, where $H_{i}(j)$ is the part covering all points closer to $O_{i}$ than to $O_{j}$ and $\overline{H_{i}(j)}$ is the remaining part of the domain space. We call $H_{i}(j)$ a half-space, and $\overline{H_{i}(j)}$ as a half-space complement. An example is shown in Fig. 4. 


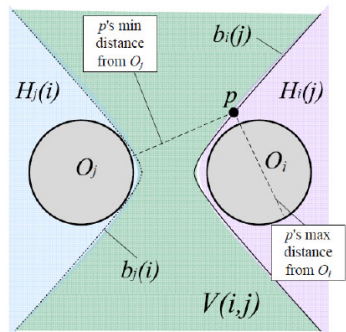

Fig. 4. u-bisector.

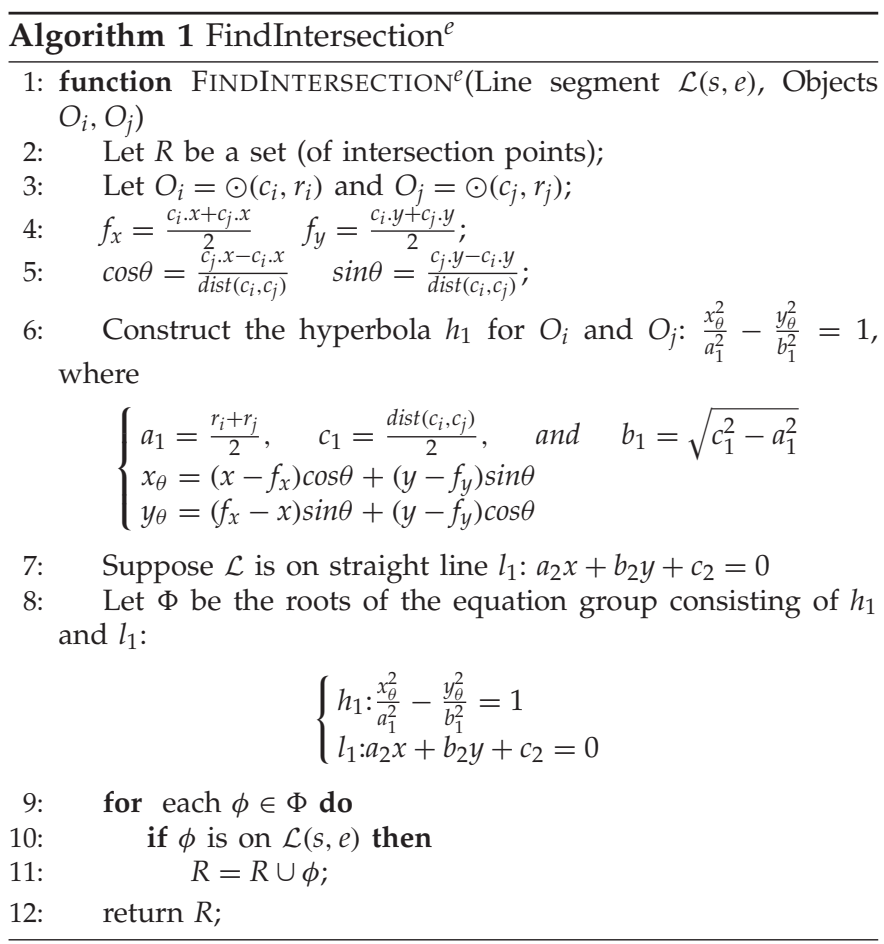

Formally, we have:

$$
\begin{aligned}
& H_{i}(j)=\left\{z \text { dist }_{\max }\left(z, O_{i}\right) \leq \operatorname{dist}_{\min }\left(z, O_{j}\right)\right\} \\
& \overline{H_{i}(j)}=\left\{z: \text { dist }_{\text {max }}\left(z, O_{i}\right)>\text { dist }_{\text {min }}\left(z, O_{j}\right)\right\} .
\end{aligned}
$$

Generally speaking, the $u$-bisector half $b_{i}(j)$ is a curve in the domain space. If a query point $q \in H_{i}(j), q$ must take $O_{i}$ as its nearest neighbor. The $u$-bisector halves $b_{i}(j)$ and $b_{j}(i)$ separate the domain into three parts, including two half-spaces $H_{i}(j)$ and $H_{j}(i)$, and a region $V(i, j)$, where

$$
V(i, j)=\overline{H_{i}(j)} \cap \overline{H_{j}(i)} .
$$

Notice that $V(i, j)=V(j, i)$. If $O_{i}$ and $O_{j}$ are degenerated into precise points, $V(i, j)$ becomes $\emptyset$ and $b_{i}(j)$ merges with $b_{j}(i)$ into a straight line.

If a query line segment is totally covered by $V(i, j), H_{i}(j)$, or $H_{j}(i)$, it does not intersect with $b_{i}(j)$ or $b_{j}(i)$. Otherwise, the intersections split the line segment into several parts. Different parts correspond to different PNNs answers, as those parts are located on different sides of $b_{i}(j)$ or $H_{j}(i)$.

For circular imprecise objects, we can derive the closed form equations of the $u$-bisectors and evaluate the analytical solution for the intersection points. The procedure to find such intersections is formalized in Algorithm 1. The number of intersections $(\Phi)$ is at most 2 , since the equation

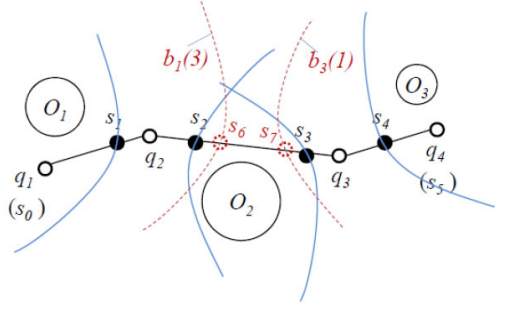

Fig. 5. Verification.

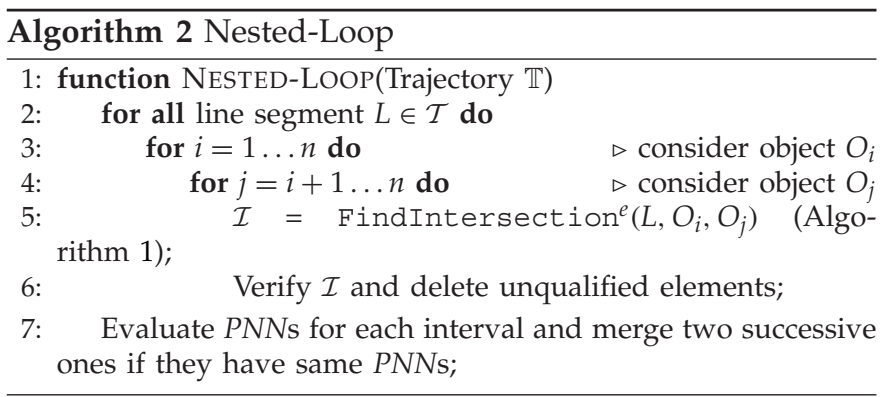

group (line 8) has at most 2 roots. Thus, Algorithm 1 can be finished in a constant time, denoted by $\beta$.

As a matter of fact, we find that the "2-intersection" fact holds for arbitrary shaped imprecise regions. For the sake of presentation, we use circular imprecise regions in following sections (Sections 2.3 to 3) and present the generalization to other shapes in Section 4.

\subsection{Evaluating TPNNQ}

In this section, we present two approaches for evaluating TPNNQ. Section 2.3.1 discusses a nested-loop approach, and Section 2.3.2 presents a more advanced approach that employs the filter-refinement paradigm.

\subsubsection{Nested-Loop Approach}

From Definition 2, the TPNNQ could be answered by deriving the turning points, which are intersections of the query trajectory and the $u$-bisectors. A $u$-bisector is constructed by a pair of objects. Given a set $O$ of $n$ objects, there can be $C_{2}^{n}$ $u$-bisectors. The Nested-Loop method (Algorithm 2) checks the intersections between the query trajectory and each of the $C_{2}^{n} u$-bisectors. The intersections are found by calling Algorithm 1 on line 5.

However, not all of the intersections are qualified as turning points. According to the definition, a turning point indicates the change of PNN answers. In Fig. $5, s_{6}$ and $s_{7}$ are not qualified as turning points, since they do not indicate such changes. $b_{1}(3)$ splits the trajectory into two parts. Thinking only of objects $O_{1}$ and $O_{3}$, one part would be definitely closer to $O_{1}$, while the other part would take both $O_{1}$ and $O_{3}$ as PNNs. In either case, $P N N Q\left(s_{6}\right)$ contains $O_{1}$. However, neither of the two cases exists, since $\mathrm{O}_{2}$ is closer. The query result for Fig. 5 is presented in Fig. 2(b). We can see that all points on segment $\left[s_{2}, s_{3}\right]$, including $s_{6}$ and $s_{7}$, take only $\mathrm{O}_{2}$ as the nearest neighbor.

In Algorithm 2, we employ a "verification" (line 6) process to exclude those unqualified intersections. Based on the discussion above, an intersection can be verified by a PNNQ. In general, given an intersection $s_{i \vdash j}=b_{i}(j) \cap \mathcal{L}$, if 


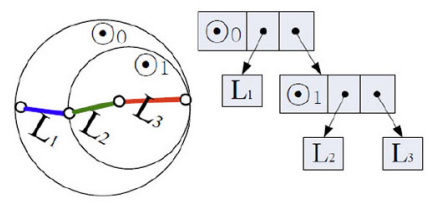

(a)

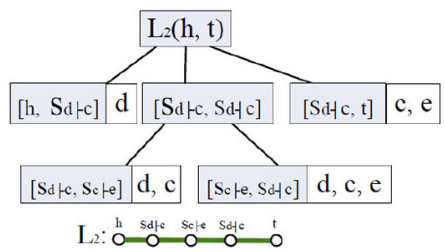

(b)
Fig. 6. Trajectory tree $\mathbb{T}(\mathcal{T})$ and Ternary tree $\Psi\left(L_{2}\right)$.

$P N N Q\left(s_{i \vdash j}\right)$ contains $O_{i}, s_{i \vdash j}$ is verified as a turning point. For example, $s_{1}$ is on $b_{1}(2)$ and $\mathrm{PNN}\left(s_{1}\right)$ contains $O_{1}$, thus $s_{1}$ is a turning point. An counter example is $s_{6}$, since $s_{6}$ is on $b_{1}(3)$, but $\mathrm{PNN}\left(s_{6}\right)$ does not contain $O_{1}$. As the PNN evaluation with a R-tree can be done in the manner of incremental nearest neighbor [6], it takes $O(\log n)$ time in practical cases [17], [18], although it could take $O(n)$ time in some rare worst cases.

Suppose $\mathcal{T}$ contains $l$ line segments, then Nested-Loop's total time complexity is $O\left(l n^{2}(\log n+\beta)\right)$. Nested-Loop is not efficient because it does not prune unqualified objects early in query evaluation but exclude them by late verifications. Next, we present a Filter-Refinement query evaluation approach that effectively prunes those unqualified objects that cannot be PNN for any point on the query trajectory.

\subsubsection{Filter-Refinement Approach}

In this section, we present a filter-refinement framework for evaluating TPNNQ. We assume an R-tree $\mathbb{R}$ is built on the imprecise objects in $O$ and it can be stored in the main memory, as the memory capabilities improve fast in recent years.

Suppose a query trajectory $\mathcal{T}$ is represented as a series of consecutive line segments, i.e., $\mathcal{T}=\left\langle L_{1}, L_{2}, \ldots, L_{l}\right\rangle$, we organize $\mathcal{T}$ using a binary trajectory tree $\mathbb{T}(\mathcal{T})$. Each binary tree node $T_{i}=\left\langle L_{1}, \ldots, L_{l^{\prime}}\right\rangle$ has two children: $T_{i}$.left $=$ $\left\langle L_{1}, \ldots, L_{\left\lfloor\frac{l^{\prime}}{2}\right\rfloor}\right\rangle$ and $T_{i}$.right $=\left\langle L_{\left\lceil\frac{l^{\prime}}{2}\right\rceil}, \ldots, L_{l^{\prime}}\right\rangle$. The trajectory tree for $\mathcal{T}=\left\langle L_{1}, L_{2}, L_{3}\right\rangle$ is shown in Fig. 6(a).

The data structure for each binary tree node $T_{i}$ is a triple: $T_{i}=\langle L, M B C$, Guard $\rangle$. Specifically, $L$ is a line segment if $T_{i}$ is a leaf-node and NULL otherwise, $M B C$ is the minimum bounded circle covering $T_{i}$ or NULL for leaf-nodes. In our algorithm, R-tree is explored gradually. Among all visited R-tree entries, Guard is the one which keeps minimum maximum distances to $T_{i}$.

The Guard entry can be either an R-tree node or an imprecise object. Note such Guard entries are not initialized until processing TPNNQ is started. Since $\mathcal{T}$ contains $l$ line segments, the trajectory tree $\mathbb{T}(\mathcal{T})$ is constructed in $O(l \log l)$ time.

The pseudo code for the filter-refinement framework is shown in Algorithm 3. It takes a trajectory tree $\mathbb{T}$ and an $\mathrm{R}$-tree $\mathbb{R}$ as input. The filtering phase is equipped with two filters. Trajectory Filter (line 3) retrieves candidate objects from $O$ such that only those objects that can be the closest objects to the query trajectory $\mathcal{T}$. All other imprecise objects are filtered due to their long distances to $\mathcal{T}$. Segment Filter (lines 4-5) further prunes unqualified candidate objects for

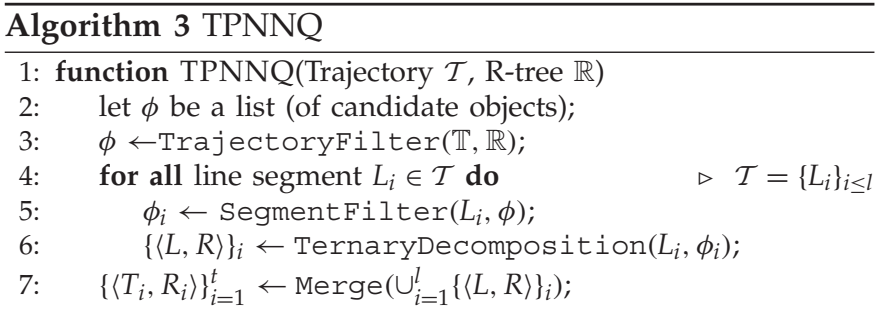

each line segment $L_{i} \in \mathcal{T}$. Our previous work [16] elaborates on how the two filters work with trees $\mathbb{T}$ and $\mathbb{R}$. We skip the details here due to the page limit.

The refinement phase evaluates all the validity intervals and turning points for each line segment in $\mathcal{T}$. This phase is encapsulated in function TernaryDecomposition(.), to be detailed in Section 3. Finally, all derived validity intervals are scanned once and consecutive ones are merged if they belong to different line segments but have the same set of PNNs (line 7).

Example of TPNNQ. Refer to Fig. 7(a). A query trajectory $\mathcal{T}=\left\{L_{1}, L_{2}, L_{3}\right\}$ is given, and an R-tree is built on imprecise objects $O=\{a, b, c, d, e, f\}$. We use trajectory filter to derive $\mathcal{T}$ 's trajectory filtering bound, as shown by shaded areas in Fig. 7(b). Objects $\{c, d, e, f\}$ overlapping with the trajectory filtering bound are taken as candidates. During the process, object $d$ is set to be $L_{2}$ 's Guard, and stored in the trajectory tree. The segment filter is applied for each line segment in $\mathcal{T}$. Taking $L_{2}$ as an example, the segment filtering bound is shown as Fig. 7(c), where $f$ is excluded from $L_{2}$ 's candidates because $f$ does not overlap with the filter bound.

In the refinement phase, we call the routine Ternary Decomposition for each line segment to derive the turning points. As shown in Fig. 7(d), we find the $u$-bisector halves $b_{d}(c)$ and $b_{c}(d)$ intersects with $L_{2}$ at $s_{d \vdash c}$ and $s_{d \dashv c}$, respectively. Thus, $L_{2}$ is split into three sub-line-segments $\left[h, s_{d \vdash c}\right]$, $\left[s_{d \vdash c}, s_{d \dashv c}\right]$, and $\left[s_{d \dashv c}, t\right]$. Meanwhile, the construction of a ternary tree $\Psi\left(L_{2}\right)$ starts accordingly, as shown in Fig. 6(b). Its root node has three children, each corresponding to a sub-line-segment. These refinement steps recur for each of

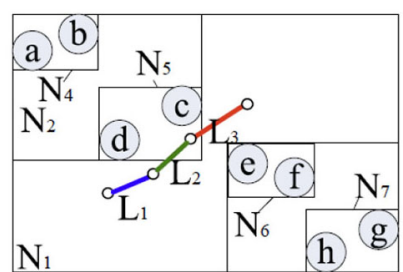

(a)

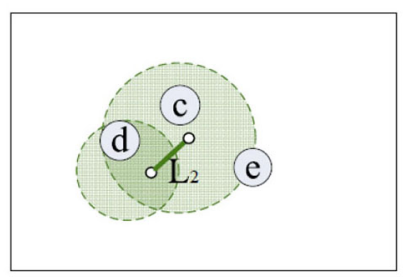

(c)

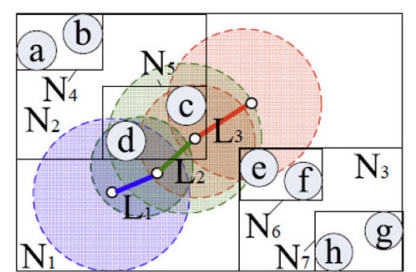

(b)

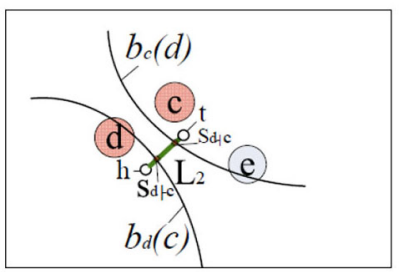

(d)
Fig. 7. TPNNQ. (a) Query input. (b) Trajectory filter. (c) Segment filter $L_{2}$. (d) Trajectory refine $L_{2}(h, t)$. 
TABLE 3

Three Cases for a Line Segment

\begin{tabular}{||c|l|l||}
\hline Case & Form & Position \\
\hline pair & {$\left[s_{i \vdash j}, s_{i \dashv j}\right]$} & $l \in V(i, j)$ \\
\hline open & {$\left[s, s_{i \vdash j}\right]$} & $\begin{array}{l}l \in H_{i}(j)\left(\text { or } l \in H_{j}(i)\right)(s(e) \text { is the } \\
\text { start(end) point of the line segment) } \\
\text { omitted }\end{array}$ \\
\hline close $\left[s_{i \dashv j}, e\right]$ & {$\left[s_{i \vdash j}, s_{i \vdash j}^{\prime}\right]$} & $l \in H_{i}(j)$ and $s_{i \vdash j}, s_{i \vdash j}^{\prime} \in b_{i}(j)$ \\
\hline
\end{tabular}

the three sub-line-segment. Finally, the process stops and a complete ternary tree $\Psi\left(L_{2}\right)$ is constructed when no further split is possible.

Note that the degree of a ternary tree node is at most 3 , since a line segment is split into at most 3 sub-line-segments (guaranteed by Theorem 2 and to be discussed in Section 4.1). Subsequently, the query result for $L_{2}$ can be fetched by traversing the leaf-nodes of $\Psi\left(L_{2}\right)$. Therefore, we have $\operatorname{TPNNQ}\left(L_{2}\right)=\left\{\left\langle\left[h, s_{d \vdash c}\right],\{d\}\right\rangle\right.$, $\left.\left\langle\left[s_{d \vdash c}, s_{c \vdash e}\right],\{c, d\}\right\rangle,\left\langle\left[s_{c \vdash e e}, s_{d \dashv c}\right],\{c, d, e\}\right\rangle,\left\langle\left[s_{d \dashv c}, t\right],\{c, e\}\right\rangle\right\}$. The results for $L_{1}$ and $L_{3}$ can be obtained likewise.

We proceed to present the refinement process that is done for each line segment in the query trajectory.

\section{Refinement Process for A Line SEgment In QUERY TRAJECTORY}

In the filter-refinement query evaluation framework, we do the refinement for each line segment $L_{i}$ in the query trajectory $\mathcal{T}$. In particular, we need to find turning points and validity intervals for a line segment $L_{i}$. We find them a recursive manner. At each iteration, we use a $u$-bisector to split the current line segment into a number of sub-linesegments. We classify the sub-line-segments into different categories and derive the specified pruning bound for each category in order to eliminate disqualified objects. The process repeats until the current intervals can not be further split. Since the current line segment is decomposed into at most 3 parts due to the at most 2 intersections, we name our algorithm ternary decomposition. Essentially, the process is equivalent to constructing a ternary tree $\Psi\left(L_{i}\right)$ for $L_{i}$.

In the sequel, we introduce categories of pruning bounds in Section 3.1. Based on that, we design the ternary decomposition algorithm in Section 3.2.

\subsection{Pruning Bounds for Three Cases}

A query line segment $L_{i}(s, e)$ can be divided by a $u$-bisector (Definition 3) into at most 3 sub-line-segments. With respect to their positions in half spaces, there are three types of subline-segments: Open Case, Pair Case, and Close Case. Refer to Fig. 4 for the sake of easy presentation. Close Case means the sub-line-segment is totally covered by $H_{i}(j)$ or $H_{j}(i)$. Open Case means the sub-line-segment is totally covered by $V(i, j)$, except that one of its endpoints is on $b_{i}(j)$ or $b_{j}(i)$. Pair Case means the sub-line-segment's two endpoints are on $b_{i}(j)$ and $b_{j}(i)$ respectively, whereas all its remaining points are in $V(i, j)$.

The three cases are formally described in Table 3.

For Pair Case and Open Case, we can derive two types of pruning bounds. Suppose the $u$-bisector between $O_{1}$ and $O_{2}$ splits the query line-segment $[s, e]$ into sub-line-segments:
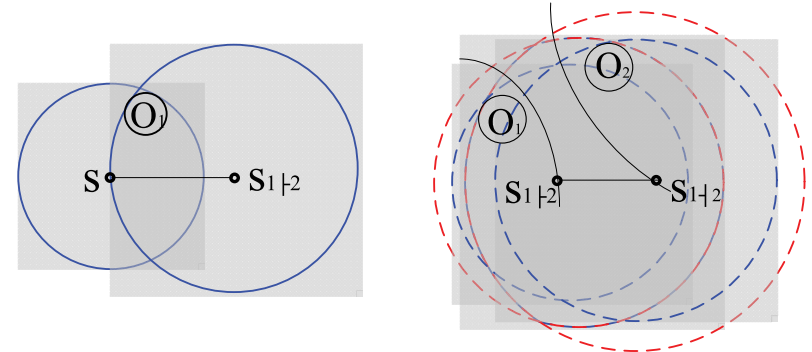

(a)

(b)

Fig. 8. Open case and pair case.

$\left[s, s_{1 \vdash 2}\right],\left[s_{1 \vdash 2}\right.$ čňs $\left.s_{1 \dashv 2}\right]$, and $\left[s_{1 \dashv 2}, e\right]$, which are of Open Case, Pair Case, and Open Case, respectively. We show the pruning bound derived for $\left[s, s_{1 \vdash 2}\right]$ and $\left[s_{1 \vdash 2}\right.$ čňs $\left.s_{1-2}\right]$ in Fig. 8(a) and (b). The bounds are highlighted by shaded areas. Note that any object $O_{i}$ beyond the bounds are safely pruned for the corresponding sub-line-segments. The pruning bound of $\left[s_{1 \dashv 2}, e\right]$ is similar to Fig. $8(\mathrm{a})$, so it is omitted.

Close Case is a special case, when a line segment has two intersections and totally inside one half-space, say $H_{i}(j)$. It could be represented by $\left[s_{i \vdash j}, s_{i \vdash j}^{\prime}\right]$, which means the two end-points are on the same $u$-bisector half $b_{i}(j)$. In this example, we know that $\left[s_{i \vdash j}, s_{i \dashv j}^{\prime}\right]$ must be in $H_{i}(j)$, so $O_{j}$ cannot be the PNN for each point inside. We design their pruning bounds in the following.

Lemma 1 (Pair Case). Given two imprecise objects $O_{i}$ and $O_{j}$, suppose their $u$-bisector $b_{i}(j)$ and $b_{j}(i)$ intersect with a straight line at $s_{i \vdash j}$ and $s_{i \dashv j} . \forall q \in\left[s_{i \vdash j} s_{i \dashv j}\right]$, an object $O_{N}$ cannot be q's PNN if $O_{N}$ has no overlap with the pruning bound $\odot\left(s_{i \vdash j}, O_{i}\right) \cup \odot\left(s_{i \dashv j}, O_{j}\right) \bigcap \odot\left(s_{i \vdash j}, O_{j}\right) \cup \odot\left(s_{i \dashv j}, O_{i}\right)$.

Lemma 2 (Open Case). Given an Open Case sub-linesegment $\left[s, s_{i \vdash j}\right], \forall q \in\left[s, s_{i \vdash j}\right]$, an object $O_{N}$ cannot be $q^{\prime} s$ $\mathrm{PNN}$, if $\mathrm{O}_{\mathrm{N}}$ has no overlap with $\odot\left(s, O_{i}\right) \cup \odot\left(s_{i \vdash j}, O_{i}\right)$.

Lemma 3 (Close Case). Given an Close Case sub-linesegment $\left[s_{i \vdash j}, s_{i \vdash j}^{\prime}\right], \forall q \in\left[s, s_{i \vdash j}^{\prime}\right]$, an object $O_{N}$ cannot be $q^{\prime} s \mathrm{PNN}$, if $\mathrm{O}_{N}$ has no overlap with $\odot\left(s_{i \vdash j}, O_{i}\right) \cup \odot\left(s_{i \vdash j}^{\prime}, O_{i}\right)$.

The proof of Lemma 1 is given in our technical report [19]. As the proofs of Lemma 2 and 3 can be easily derived from Lemma 8 (in Appendix), they are omitted due to page limit.

The Pair Case can also be considered as the union of two Open Cases. For example, a Pair Case $\left[s_{i \vdash j}, s_{i \dashv j}\right]$ is equivalent to the overlap part of $\left[s, s_{i \dashv j}\right]$ and $\left[s_{i \vdash j}, e\right]$. Moreover, the Close Case can be viewed as the union of $\left[s, s_{i-j}^{\prime}\right]$ and $\left[s_{i \vdash j}, e\right]$. The three cases and their combinations cover all possibilities for each piece (validity interval) of a query line segment $L_{i}$. After the ternary tree $\Psi\left(L_{i}\right)$ is constructed for $L_{i}$, we can derive the pruning bound of a validity interval. It is the intersection of all its ascender nodes' pruning bounds in the ternary tree $\Psi$.

\subsection{Ternary Decomposition}

The ternary decomposition constructs the ternary tree $\Psi$ in an iterative manner, as shown in Algorithm 4. At each iteration, we select two objects from the current candidate set $\phi_{c u r}$ as seeds to divide the current line-segment $L_{c u r}$ into two or three pieces. To split $L_{c u r}$, we have to evaluate a 


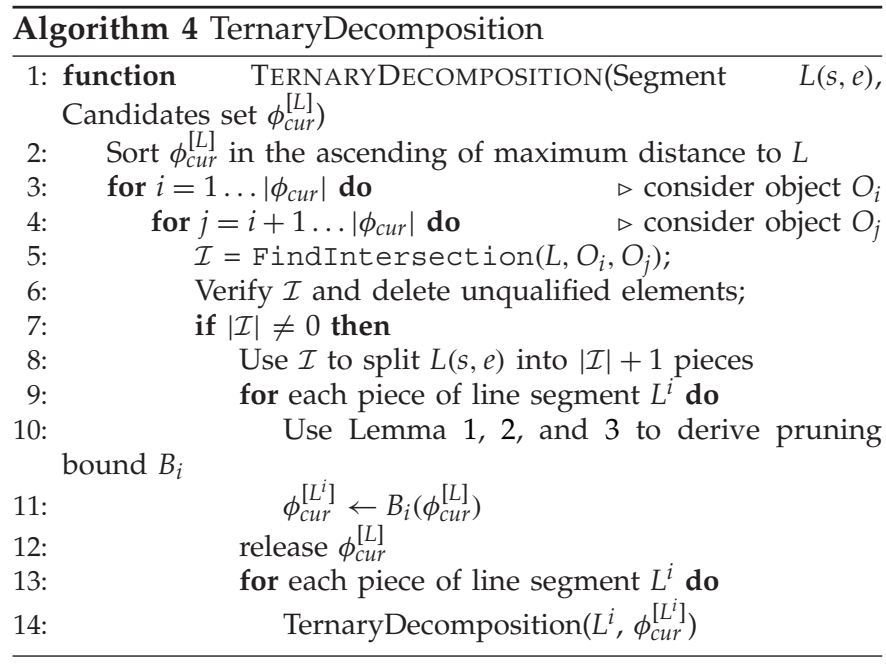

feasible $u$-bisector, whose intersections with $L_{c u r} i$ are turning points. Then, to find the $u$-bisector, we might have to try $\frac{C(C-1)}{2}$ pairs of objects, where $C=\left|\phi_{\text {cur }}\right|$. In fact, the object with the minimum maximum distance to $L_{c u r}$, say $O_{1}$, must be one PNN. The correctness is shown in Lemma 4.

Lemma 4. If $S=\left\{O_{1}, O_{2}, \ldots\right\}$ are sorted in the ascending order of the maximum distance to the line segment $L$, then $O_{1} \in$ $T P N N Q(L)$.

Accordingly, the turning points on $L_{c u r}$ are often derived by $O_{1}$ and another object among the $C$ candidates. Therefore, the candidates are sorted first in the ternary decomposition. After that, $L_{c u r}$ is split into 2 (or 3 ) pieces (or children). For $L_{c u r}$ 's children $L^{i}$, we derive a pruning bound $B_{i}$ for $L^{i}$ and select a subset of candidates from $\phi_{\text {cur }}$ (lines 9 to 12). Notice that for each leaf-node $L^{i}$ of the ternary tree $\Psi(L(s, e)), L^{i}$ 's two endpoints must be $s, e$, or the turning points on $L$. If we traverse $\Psi$ in the pre-order manner, any two successively visited leaf-nodes are the successively connected validity intervals in L. Suppose we have $m$ turning points, we would have $m+1$ validity intervals, which corresponds to $m+1 \Psi^{\prime}$ s leaf-nodes. Algorithm 4 stops when any pair of objects in $\phi_{c u r}^{[L]}$ does not further split $L$.

The complexity of ternary decomposition depends on the size of the turning points in the final result. A ternary tree node $T_{i}$ splits only if one or two intersections are found in $T_{i}$ 's line segment. If no intersections are found in its line segment, $T_{i}$ becomes a leaf-node. Given the final answer containing $m$ turning points, there would be at most $2 m$ nodes in the ternary tree $\Psi(\mathcal{T})$. At least, there are $\lceil 1.5 m\rceil$ nodes. So Algorithm 4 will be called $(1.5 m, 2 m$ ] times. suppose that line 5 in Algorithm 4 is done in time $\beta$ and line 6 is in $O(\log C)$, where $C$ is the number of candidate objects returned by the filtering phase in Algorithm 3. As a result, the complexity of ternary decomposition is $O\left(m C^{2}(\log C+\beta)\right)$.

\section{Supporting Arbitrary Shapes of IMPRECISE REGIONS OF OBJECTS}

So far we have presented our solution for TPNNQ where all imprecise objects have circular imprecise regions. It is however possible that imprecise objects take arbitrary shapes of imprecise regions, as illustrated in Fig. 1. To handle different shapes, an intuitive way is to enclose an object by a minimum bounding circle ( $M B C$ in short), and then evaluate the query on the $M B C$ s. This makes sense when the imprecise regions can be well represented by MBCs. Otherwise, MBC can introduce considerable dead space, and thus cause many false positives that degrade the query result quality. Hence, it is desirable to have a solution that is more general, reliable, and deployable.

As a matter of fact, the proposed techniques in previous sections can be generalized to arbitrary imprecise region shapes. In particular, to apply the derived techniques (Lemma 123 and 4), we need to instantiate dist $t_{\max }$ (.) (or dist $\left._{\text {min }}().\right)$ for each specific type of shapes. In addition, we need to consider two important aspects. First, the "2-intersection" fact should hold for other arbitrary. We need to guarantee this in order to make the Ternary Decomposition (Section 3) still work. Second, the $u$-bisector's form for arbitrary shaped imprecise regions can be complex. We need to find the turning points (recall Algorithm 1) for the complex case where the $u$-bisector's math representation is not available.

\subsection{Theories about the $u$-bisector}

One important geometric property about the $u$-bisector half $b_{i}(j)$ is: half space $H_{i}(j)$ is convex. This property holds even if the imprecise region's shape is concave and irregular. Next, we prove the property formally.

Theorem 1 (Half Space Convexity). Given two imprecise objects $O_{i}$ and $O_{j}$, the half space $H_{i}(j)$ enclosed by the $u$-bisector half $b_{i}(j)$ is convex.

Proof. According to Midpoint Convexity Theorem [20], if two arbitrary points $s, e \in H_{i}(j)$, whose midpoint $m=\frac{s+e}{2}$ satisfies $m \in H_{i}(j)$, then $H_{i}(j)$ is convex.

Suppose that two precise points $p_{i} \in O_{i}$ and $p_{j} \in O_{j}$ satisfy:

$$
\left\{\begin{array}{l}
\operatorname{dist}_{\text {max }}\left(m, O_{i}\right)=\operatorname{dist}\left(m, p_{i}\right) \\
\operatorname{dist}_{\text {min }}\left(m, O_{j}\right)=\operatorname{dist}\left(m, p_{j}\right)
\end{array}\right.
$$

Also,

$$
s \in H_{i}(j) \Rightarrow \operatorname{dist}\left(s, p_{i}\right) \leq \operatorname{dist}\left(s, p_{j}\right) .
$$

Similarly,

$$
\operatorname{dist}\left(e, p_{i}\right) \leq \operatorname{dist}\left(e, p_{j}\right) \text {. }
$$

Applying Lemma 10 (see Appendix) to Equations 2 and 3 , we have:

$$
\begin{array}{r}
\operatorname{dist}\left(m, p_{i}\right) \leq \operatorname{dist}\left(m, p_{j}\right) \\
\Rightarrow \operatorname{dist}_{\text {max }}\left(m, O_{i}\right) \leq \operatorname{dist}_{\text {min }}\left(m, O_{j}\right)(\text { Equation } 1) \\
\Rightarrow m \in H_{i}(j) \Rightarrow H_{i}(j) \text { is convex. }
\end{array}
$$

The theorem is thus proved.

Based on $H \_i(j)$ 's convex property, a line segment $\mathcal{L}$ could have at most two intersections with $b \_i(j)$. Formally,

Lemma 5. Given two imprecise objects $O_{-} i$ and $O_{-} j$, a line segment $\mathcal{L}(s, e)$ has at most two intersection points with the $u$-bisector half $b_{-} i(j)$.

Since $H_{i}(j)$ is convex, its intersection with line segment $\mathcal{L}$ is $l=\mathcal{L} \cap H_{i}(j)$, which must also be convex. Since $l$ is 
TABLE 4

Four Types of a Query Line Segment

\begin{tabular}{||c|l||}
\hline $\begin{array}{c}\text { Intersection } \\
\text { count }\end{array}$ & Condition \\
\hline \multirow{2}{*}{0} & $\mathrm{~A}: \mathcal{L}(s, e) \in H_{i}(j)$ or $\mathcal{L}(s, e) \in H_{j}(i) \quad$ (Lemma 6) \\
\cline { 2 - 3 } & $\mathrm{B}: \mathcal{L}(s, e) \in V(i, j) \quad($ Lemma 7) \\
\hline 1 & $s(e) \in H_{i}(j) / H_{j}(i)$ and $e(s) \in V(i, j)$ \\
\hline 2 & $\mathrm{~A}: s \in H_{i}(j) \wedge e \in H_{j}(i)$ or $s \in H_{j}(i) \wedge e \in H_{i}(j)$ \\
\cline { 2 - 3 } & $\mathrm{B}: s, e \in V(i, j) \wedge \exists q \in \mathcal{L}, q \in H_{i}(j)$ or $H_{j}(i)$ \\
\hline Unknown & can be either 0 -B or 2 -B \\
\hline
\end{tabular}

also a part of $\mathcal{L}, l$ is a line segment or $\emptyset$. If $l=\emptyset, l$ has no intersections with $b_{i}(j)$. Otherwise, $l$ has at most two intersections with the $u$-bisector half $b_{i}(j)$, whereas $l$ 's two end points are on $H_{i}(j)$ 's boundary.

Likewise, Theorem 1 and Lemma 5 hold for the $H_{j}(i)$ and $b_{j}(i)$. Next, we show a more interesting property about the number of intersections between a line segment $\mathcal{L}$ and the $u$-bisector as a whole.

Theorem 2 (Two-intersection Theorem). Given two imprecise objects $O_{i}$ and $O_{j}$, a line segment $\mathcal{L}$ has at most two intersections with the $u$-bisector that consists of $b_{i}(j)$ and $b_{j}(i)$.

Proof. It is sufficient to show: if $\mathcal{L}$ intersects with $b_{i}(j)$ at two points, $\mathcal{L} \cap H_{j}(i)=\emptyset$. In other words, we need to prove for an arbitrary point $t \in \mathcal{L} \wedge t \notin H_{i}(j), t \notin H_{j}(i)$.

For circular imprecise regions, the theorem is true according to Lemma 11 (see Appendix). For non-circular imprecise regions, we apply the site decomposition idea [21] to decompose $O_{i}$ and $O_{j}$ into two sets of circles $P$ and $Q$. The circles in $P$ or $Q$ may be of different sizes and overlap. An overall half-space $H_{j}(i)$ is the intersection of all half-spaces $H_{j(q)}(i(p))$ where $p \in P$ and $q \in Q$ (see Lemma 9 in Appendix).

Let $u_{i}=\left\{u_{i(p)}\right\}_{p \in P}$ and $u_{j}=\left\{u_{j(q)}\right\}_{q \in Q}$. For each pair of $u_{i}(p)$ and $u_{j}(q)$, we can prove $t \notin H_{j(q)}(i(p))$ according to Lemma 11. Hence, we have:

$$
\begin{gathered}
\forall q \in Q \quad \forall p \in P, t \notin H_{j(q)}(i(p)) \Rightarrow \\
t \notin \cap_{p \in P \wedge q \in Q} H_{j(q)}(i(p)) \Rightarrow t \notin H_{j}(i) .
\end{gathered}
$$

Thus, the theorem is true.

Theorem 2 tells that a $u$-bisector can split the query line segment into 3 sub-line segments at most, no matter what shapes the imprecise regions of the two objects have and how complex the form of the $u$-bisector is. Supported by Theorem 2, we proceed to show how to find intersections when arbitrary imprecise region shapes are involved.

\subsection{Finding Intersections Involving Arbitrary Imprecise Region Shapes}

For arbitrary imprecise regions, whose $u$-bisector's mathematical representation is not available, we design an approximated method to find the intersections. The intuition of doing that is to decompose the line segment into smaller pieces in order to approach the intersections. The decomposition stops if intersections are found or the current line segment contains no intersections. Otherwise, as the decomposition continues, the intersection will be infinitely approached. To do that, we have to: 1) detect whether the current line segment contains intersections; 2) determine whether the approximation is sufficiently accurate. For 1),

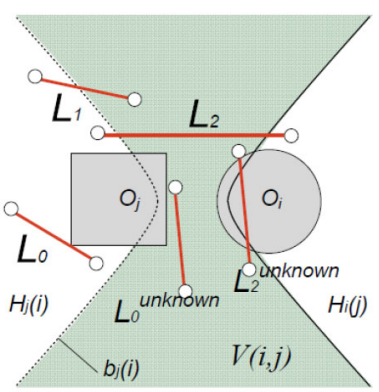

Fig. 9. Types of a line segment.

we use Table 4 to list all possible cases of intersections on a line segment, as well as their judging criteria. For 2), we use a precision parameter $T_{\epsilon}$. The method is described in more detail below.

Given a line segment and two objects $O_{i}$ and $O_{j}$ 's $u$ bisector, there can be at most two intersections, as revealed by Theorem 2 . We thus classify the line segment into 4 different categories according to the number of intersections, as shown in Table 4. Different cases correspond to different conditions. Referring to the example shown in Fig. 9, $L_{1}$ 's two endpoints are located in $H_{j}(i)$ and $V(i, j)$, so $L_{1}$ belongs to type 1 . Also, $L_{0}$ and $L_{2}$ belong to type 0 -A and 2A respectively, according to the conditions listed in Table 4. However, $L_{0}^{\text {unknown }}$ and $L_{2}^{\text {unknown }}$ are two "undetermined" cases. If we only know that one line segment's endpoints are in $V(i, j)$, we can not tell if it is of type 0-B (e.g., $L_{0}^{\text {unknown }}$ ) or 2-B (e.g., $\left.L_{2}^{\text {unknown }}\right)$. We use $L^{\text {unkown }}$ to represent the case that a line segment's two endpoints are in $V(i, j)$. Thus, it is hard to detect which type the $L^{\text {unkown }}$ belongs to. We have developed Lemma 7 for type 0-B. Nevertheless, not all cases in type 0-B can be captured. For "undermined" types, we can recursively decompose the line segments, until all the sub-line segments can be classified.

Lemma 6. A line segment $\mathcal{L}$ is in the region $V(i, j)$ iff:

$$
\begin{aligned}
\forall p \in \mathcal{L}, \operatorname{dist}_{\text {max }}\left(p, O_{i}\right)>\operatorname{dist}_{\text {min }}\left(p, O_{j}\right) \\
\wedge \operatorname{dist}_{\text {max }}\left(p, O_{j}\right)>\operatorname{dist}_{\text {min }}\left(p, O_{i}\right) .
\end{aligned}
$$

Proof.

According to the definition of half space:

$$
\begin{aligned}
p & \in V(i, j) \Leftrightarrow p \notin H_{i}(j) \wedge p \notin H_{j}(i) \\
& \Leftrightarrow \operatorname{dist}_{\max }\left(p, O_{i}\right)>\operatorname{dist}_{\text {min }}\left(p, O_{j}\right) \\
& \wedge \operatorname{dist}_{\text {max }}\left(p, O_{j}\right)>\operatorname{dist}_{\text {min }}\left(p, O_{i}\right) .
\end{aligned}
$$

Thus,

$$
\begin{array}{r}
\mathcal{L} \in V(i, j) \Leftrightarrow \\
\forall p \in \mathcal{L}, \operatorname{dist}_{\text {max }}\left(p, O_{i}\right)>\operatorname{dist}_{\text {min }}\left(p, O_{j}\right) \wedge \\
\operatorname{dist}_{\text {max }}\left(p, O_{j}\right)>\text { dist }_{\text {min }}\left(p, O_{i}\right) .
\end{array}
$$

Lemma 7. A line segment $\mathcal{L}$ is in the region $V(i, j)$ if:

$$
\begin{gathered}
\operatorname{dist}_{\text {max }}\left(m, O_{i}\right)>\operatorname{dist}_{\text {min }}\left(m, O_{j}\right)+\operatorname{length}(\mathcal{L}) \\
\wedge \operatorname{dist}_{\text {max }}\left(m, O_{j}\right)>\operatorname{dist}_{\text {min }}\left(m, O_{i}\right)+\operatorname{length}(\mathcal{L}),
\end{gathered}
$$

where $m$ is the middle point of $\mathcal{L}$. 


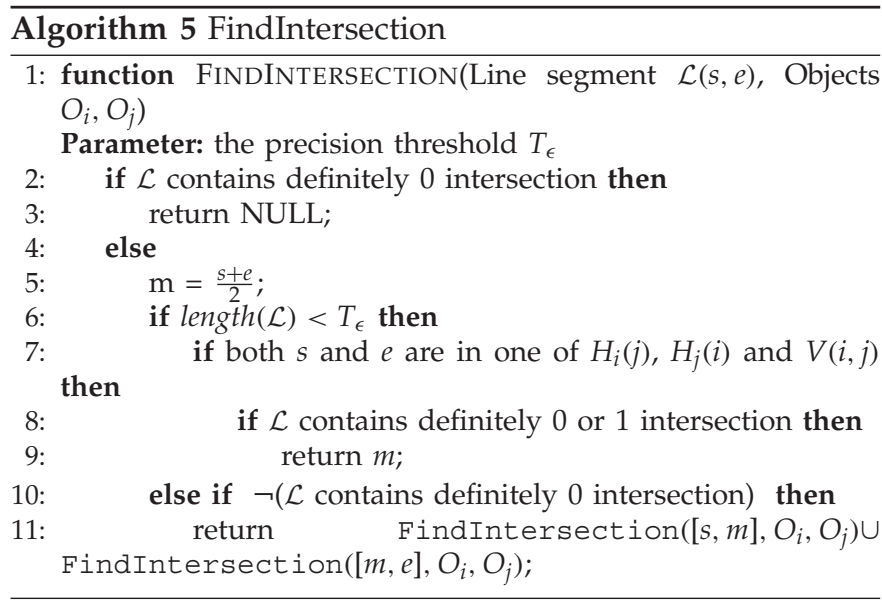

Proof. Let $p$ be an arbitrary point on the line segment $\mathcal{L}, x$ be any location in $O_{i}$, and $r_{m}=\frac{\text { length }(\mathcal{L})}{2}$.

$$
\begin{gathered}
\operatorname{dist}_{\text {max }}\left(m, O_{i}\right)>\operatorname{dist}_{\text {min }}\left(m, O_{j}\right)+\operatorname{length}(\mathcal{L}) \Rightarrow \\
\operatorname{dist}_{\text {max }}\left(m, O_{i}\right)-r_{m}>\operatorname{dist}_{\text {min }}\left(m, O_{j}\right)+r_{m} .
\end{gathered}
$$

We consider the left-hand side of Equation 4 first. Let $y$ be a point of $O_{i}$ such that $\operatorname{dist}_{\max }\left(m, O_{i}\right)=\operatorname{dist}(m, y)$. By triangle inequality, we have $\operatorname{dist}(p, y) \geq$ $\operatorname{dist}(m, y)-\operatorname{dist}(p, m)=\operatorname{dist}_{m a x}\left(m, O_{i}\right)-\operatorname{dist}(p, m)$. As $m$ is the middle point of $\mathcal{L}$, we have $r_{m}=\frac{\text { length }(\mathcal{L})}{2} \geq \operatorname{dist}(p, m)$. We also have $\operatorname{dist}_{\text {max }}\left(p, O_{i}\right) \geq \operatorname{dist}(p, y)$. From these three inequalities, we have

$$
\operatorname{dist}_{\max }\left(p, O_{i}\right) \geq \operatorname{dist}_{\max }\left(m, O_{i}\right)-r_{m} .
$$

Likewise, for the right-hand side of Equation 4, we have:

$$
\operatorname{dist}_{\min }\left(m, O_{j}\right)+r_{m} \geq \operatorname{dist}_{\min }\left(p, O_{j}\right) .
$$

Considering Equations 4, 5 and 6 altogether, we have:

$$
\forall p \in \mathcal{L}, \operatorname{dist}_{\text {max }}\left(p, O_{i}\right)>\operatorname{dist}_{\text {min }}\left(p, O_{j}\right) .
$$

Similarly, we can prove

$$
\forall p \in \mathcal{L}, \operatorname{dist}_{\text {max }}\left(p, O_{j}\right)>\operatorname{dist}_{\text {min }}\left(p, O_{i}\right) .
$$

According to Lemma 6, Equations 7 and 8 are sufficient to show $\mathcal{L}$ is in the region $V(i, j)$.

Based on the four types of a line segment, we compute the intersection points approximately using Algorithm 5. The idea of the approximation is to recursively split the query line segment until the current line segment, which contains the type 1 intersection, is shorter than the precision threshold $T_{\epsilon}$. We thus return the middle point of the line segment as an intersection.

During the decomposition, we classify the line segments into 4 types following Table 4 . If the current line segment is of type 1 or 2 , it is decomposed for evaluating intersections. If it is of type 0 , the branch is stopped. Otherwise, it is of type Unknown, the line segment is also decomposed for clarification. The complexity of Algorithm 5 is $O\left(\log _{T_{\epsilon}}|L|\right)$.

For common shapes, such as line segments, circle, rectangles, we can derive the closed form equation for dist $t_{\text {min }}$ and dist max $_{\text {and }}$ substitute them into Algorithm 5. In real

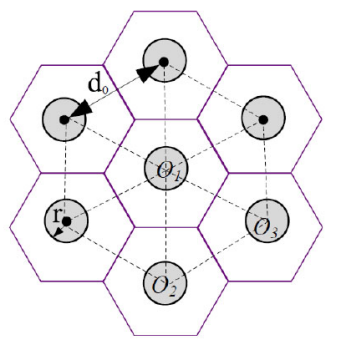

Fig. 10. Hexagonal model.

applications, objects can have complex shapes. An iceberg floating on the sea could have an irregular contour. Or the location of a vehicle moving on the road network could be summarized as a polyline. However, if the complex shaped object can be represented by the combination of simple shapes (e.g., circles, line segments, rectangles), our solution can be replanted. For example, the moving object in a road network can be represented by a polyline, which consists of a set of line-segments. Or the iceberg can be decomposed into a set of circles or rectangles. In general, if a complex shaped object $O$ can be represented by a set of simple shapes $\left\{m_{i}\right\}$, the minimum and maximum distances between a query point $q$ to $O$ can be calculated by:

$$
\left\{\begin{array}{l}
\operatorname{dist}_{\text {min }}(q, O)=\min _{i}\left\{\operatorname{dist}_{\text {min }}\left(q, m_{i}\right)\right\} \\
\operatorname{dist}_{\max }(q, O)=\max _{i}\left\{\operatorname{dist}_{\max }\left(q, m_{i}\right)\right\} .
\end{array}\right.
$$

By substituting those equations into Table 4 and Algorithm 5, our solution can be extended to support those complex shaped imprecise regions, even if they are concave. The correctness can be guaranteed by Theorem 1 and 2 .

\section{Selectivity Estimation for TPNNQ}

Accurate selectivity estimation is crucial for query processing in database systems. In many mobile subscriptions, it is important to estimate the size of the data to be transmitted (e.g. $\phi$ in Algorithm 3) from the LBS server to the client, because that means how much money the subscriber needs to pay. In some settings, the LBS server can also decide to stop the processing if it finds out the results size is higher than the client's upper limit that is indicated by his subscription.

In this section, we study selectivity estimation for $T P N N Q$. We start from the simplest case where the query is a point (Section 5.1). Further, we extend it to query linesegments (Section 5.2) and query trajectories (Section 5.3). We consider the hexagonal lattice model [22], [23], as shown in Fig. 10, where each object has six neighbors whose centers are equidistant from each other, with distance $d_{0}{ }^{1}$. We assume that the imprecise regions are equal-sized and circular shaped with a radius of $r$.

\subsection{Result Size Analysis for Query Point}

To derive the number of possible nearest neighbors for a given query point $q$, we need to estimate the minimum

1. The centers of uncertainty regions form the vertices of $n$ hexagons, each of which has an area of $\frac{\sqrt{3} d_{0}^{2}}{2}$. Since $|D|=n \times \frac{\sqrt{3} d_{0}^{2}}{2}$, $d_{0}=\sqrt{\frac{2|D|}{\sqrt{3} n}}$. 


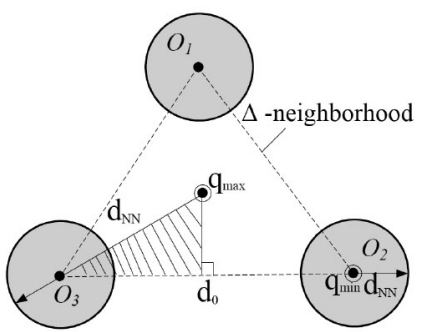

Fig. 11. $\Delta$-neighborhood.

maximum distance from $q$ to all imprecise objects. We use $d_{N N}$ to denote that distance. Subsequently, the search region of $P N N(q)$ is the circle centered at $q$ with radius $d_{N N}$. Objects that overlap with $\odot\left(q, d_{N N}\right)$ are qualified as $q$ 's possible nearest neighbors [6].

If we connect the centers of two adjacent objects, the domain would be triangulated by dashed lines as shown in Fig. 10. Given query point $q$, it must be resided in a triangle. We denote it as $\Delta$-neighborhood, which consists of three objects, as shown in Fig. 11. Among the three, there must be one object having the minimum maximum distance $d_{N N}$ to $q$, since these three objects are closer than others outside. Different locations in $\Delta$-neighborhood correspond to different $d_{N N}$ s. In Fig. $11, q_{\text {min }}$ 's $d_{N N}$ is $O_{2}$ 's radius, and $q_{\max }$ 's $d_{N N}$ is the distance from $q_{\max }$ to $\mathrm{O}_{3}$ 's center plus $\mathrm{O}_{3}$ 's radius. Since $d_{N N}$ is changing over $q$ 's locations, the number of PNNs also varies. If we define the density $\rho$ as the number of objects over a unit area, then the number of PNNs can be measured by the density times the area of the search region. Thus, we can get the expected number of PNNs. We first derive the $E(|P N N|)$ for the shaded area (in Fig. 11) denoted as $\Delta_{\text {shaded, }}$, and repeat 6 times to cover the entire $\Delta$-neighborhood.

$$
\begin{array}{r}
E\left(|P N N(q)|_{q \in \Delta \text {-neighborhood })}\right. \\
=\frac{\int_{q \in \Delta}|P N N(q)| d q}{\mid \Delta \text {-neighborhood } \mid}=\frac{6 \cdot \int_{q \in \Delta_{\text {shaded }}}|P N N(q)| d q}{6 \cdot\left|\Delta_{\text {shaded }}\right|} \\
=\frac{6 \cdot \int_{q \in \Delta_{\text {shaded }}} \rho \pi d_{N N}^{2} d q}{6 \cdot\left|\Delta_{\text {shaded }}\right|} \\
=\frac{\int_{0}^{\frac{d_{0}}{2}} \int_{0}^{\frac{x}{\sqrt{3}}} \rho \pi\left(\sqrt{x^{2}+y^{2}}+r\right)^{2} d y d x}{\frac{1}{2} \cdot \frac{d_{0}}{2} \cdot \frac{d_{0}}{2 \sqrt{3}}} \\
=\rho \pi\left[\frac{\sqrt{3} d_{0}\left(24 r+5 \sqrt{3} d_{0}+18 r \log _{2} \sqrt{3}\right)}{108}+r^{2}\right] .
\end{array}
$$

Also, since $\rho$ and $\pi$ are independent, by extracting them we can derive $E\left(d_{N N}\right)$ :

$$
\begin{aligned}
& \rho \pi E^{2}\left(d_{N N}\right)=E(|P N N(q)|) \\
& E\left(d_{N N}\right)=\sqrt{\frac{E(|P N N(q)|)}{\rho \pi} .}
\end{aligned}
$$

In the sequel, we simply use $d_{N N}$ to represent $E\left(d_{N N}\right)$.

\subsection{Result Size Analysis for Query Line Segment}

If the query is a line-segment instead of a point, the search region would be the union of search regions for all points on the line-segment. We show an example of the search

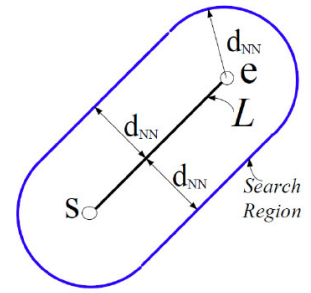

Fig. 12. $|P N N(\mathcal{L})|$.

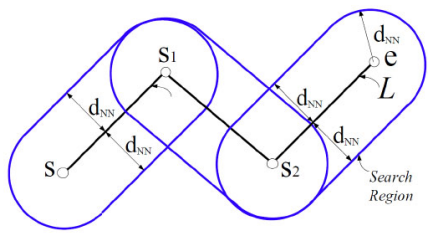

Fig. 13. $|P N N(\mathcal{T})|$.

region of line-segment $\mathcal{L}$ in Fig. 12. The number of $\mathcal{L}^{\prime} \mathrm{s} P N N \mathrm{~s}$ can be calculated as the product of density $\rho$ and the search region's area. $d_{N N}$ can be calculated by Equation 9 .

$$
E(|P N N(\mathcal{L})|)=\rho \pi\left(2 \cdot d_{N N} \cdot|\mathcal{L}|+\pi \cdot d_{N N}^{2}\right) .
$$

\subsection{Result Size Analysis for Query Trajectory}

Now we extend the estimation from query line-segments to query trajectories. Suppose trajectory $\mathcal{T}$ is represented by $\left\{\mathcal{L}_{1}, \ldots, \mathcal{L}_{l}\right\}$, where successive line-segments $\mathcal{L}_{i}$ and $\mathcal{L}_{i+1}$ are connected by point $s_{i}$. Then, $\mathcal{T}^{\prime}$ s search region equals to the union of all $\mathcal{L}_{i}$ 's search regions, as shown in Fig. 13. The union could be well approximated by the summation of all line-segments $\left(\left\{L_{i}\right\}\right)^{\prime}$ s search regions subtracting all connecting points $\left(\left\{s_{i}\right\}\right)^{\prime}$ 's search regions.

$$
\begin{array}{r}
E(|P N N(\mathcal{T})|) \approx \\
\sum_{\mathcal{L}_{i} \in \mathcal{T}} E\left(\left|P N N\left(\mathcal{L}_{i}\right)\right|\right)-\sum_{s_{i} \in \mathcal{T}} E\left(\left|P N N\left(s_{i}\right)\right|\right) .
\end{array}
$$

The analysis above can be extended to other object distributions as follows. We apply an equal-sized histogram which splits the domain into $m \times m$ squares. For each square $s$, we assume the objects are uniformly distributed inside. We count the number of objects $N(s)$ of square $s$. Thus, the density $\rho(s)$ of $s$ is collected by $\frac{N(s)}{|D| /(m \times m)}$. We take the average density $\bar{\rho}$ for all squares overlapping with the query trajectory $\mathcal{T}^{2}$, and substitute them into Equation 11 to get the estimation.

\section{EXPERIMENTAL Evaluation}

In this section we report on the experimental results on different datasets. Section 6.1 describes the relevant settings. Section 6.2 gives a metric to measure to quality of query results. Section 6.3 presents the experimental results.

\subsection{Experimental Settings}

Queries. The query trajectories are generated by Brinkhoff's network-based mobile data generator ${ }^{3}$. The

2. Other parameters such as $\overline{d_{0}}$ and $\bar{r}$ are obtained similarly.

3. http:/ /iapg.jade-hs.de/personen/brinkhoff/generator/ 
trajectory represents movements over the road-network of Oldenburg city in Germany. We normalize them into $10 \mathrm{~K}$ $\times 10 \mathrm{~K}$ space. By default, the length of trajectory is 500 units. Each reported value is the average of 20 trajectory query runs.

Imprecise Objects. We use four real datasets of geographical objects in Germany and U.S. ${ }^{4}$, namely germany, LB, stream and block with 30K, 50K, 199K, 550K spatial objects, respectively. We also construct the $\mathrm{MBC}$ for each object and get 4 other datasets with circular imprecise regions ${ }^{5}$. We use stream as the default dataset. Datasets are normalized to the same domain as queries.

To index imprecise regions, we use a packed $R^{*}$-tree [24]. The page size of $\mathrm{R}$-tree is set to $4 \mathrm{~K}$-byte, and the fanout is 50. The entire $R^{*}$-tree is accommodated in the main memory.

All our programs were implemented in $\mathrm{C}++$ and tested on a Core2 Duo 2.83GHz PC enabled by MS Windows 7 Enterprise.

\subsection{Query Result Quality Metric}

As TPNNQ queries over imprecise objects, it is interesting to measure the query result quality. We adopt an Error function based on the Jaccard Distance [25], which measures the similarity between two sets. Recall that the query result of TPNNQ is a set of tuples $\left\{\left\langle T_{i}, R_{i}\right\rangle\right\}$. It can be transformed into the PNNs for every point on the query trajectory $\mathcal{T}$, i.e., $\{\langle q, P N N Q(q)\rangle\}_{q \in \mathcal{T}}$. Let $R^{*}(q)=P N N Q(q)$ be the groundtruth query result for a point $q$. We use $R^{A}(q)$ to represent the $P N N$ s returned by algorithm $A$ for the point $q$. The Error for algorithm $A$ on query $\mathcal{T}$ is:

$$
\operatorname{Error}(\mathcal{T}, A)=\frac{1}{|\mathcal{T}|} \int_{q \in \mathcal{T}} 1-\frac{R^{*}(q) \cap R^{A}(q)}{R^{*}(q) \cup R^{A}(q)} d q
$$

Here, $|\mathcal{T}|$ is the total length of trajectory $\mathcal{T}$. If $\mathcal{T}$ is represented by a set of line segments $\mathcal{T}=\left\{L_{i}\right\}_{i=1}^{t}$, the total length $|\mathcal{T}|=\sum_{i=1}^{t}\left|L_{i}\right|$.

Equation 12 captures the effect of false positives and false negatives as well. There is a false positive when $R^{A}(q)$ contains an extra item not found in $R^{*}(q)$. There is a false negative when an item of $R^{*}(q)$ is missing from $R^{A}(q)$. For a perfect method with no false positives and false negatives, the two terms $R^{*}(q)$ and $R^{A}(q)$ are the same, so the integration value is 0 . In implementation, it is not feasible to check all $q \in \mathcal{T}$ to calculate Equation 12. Instead, we use the Monte-carlo method with very large sampling rate (by setting the sampling interval to be $10 e^{-5}$ unit) to accurately calculate the integration.

In summary, the error score is a value between 0 and 1 . The smaller an error score is, the more accurate the result is. On the other hand, if a method has many extra or missing results, it acquires a high error score.

\subsection{Performance Results}

The query performance is evaluated by two metrics: efficiency and quality. The efficiency is measured by counting the clock time. The quality is measured by the error score

4. http://www.rtreeportal.org/

5. We handle other shaped imprecise regions in Section 6.3.4.

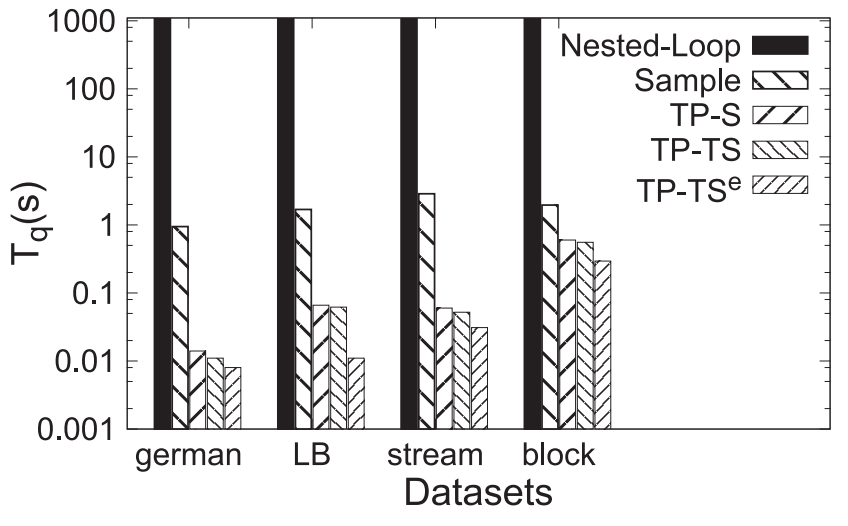

Fig. 14. $T_{q}(\mathrm{~s})$ vs. datasets.

defined in Section 6.2. To evaluate the filter-refinement query evaluation framework (Algorithm 3), we list several competitors: Nested-Loop, Sample, TP-S, TP-TS, and TP-TS ${ }^{e}$. The suffixes $T$ and $S$ refer to Trajectory Filter and Segment Filter, respectively. Nested-Loop does not use any filter; TP-S does not use Trajectory Filter; TP-TS and TP-TS (Algorithm 3) use all the filtering and refinement techniques. Sample draws a set of uniform sampling points $\{q\}$ from $\mathcal{T}$. Then, for all $q, P N N Q(q)$ is evaluated. The sampling interval, denoted by $\epsilon$, is set to 0.1 unit by default ${ }^{6}$.

As discussed, we either use Findintersection ${ }^{e}$ (Algorithm 1) to find exact turning points or FindIntersection (Algorithm 5) to find approximated turning points. The superscript $e$ indicates the exact intersection calculation. So, TP-TS ${ }^{e}$ derives exact turning points for circular regions, while TP-TS calculates approximate turning points for arbitrary shaped regions. For FindIntersection ${ }^{e}$, we call GSL Library $^{7}$ to get the analytical solution. For Findintersection, the default $T_{\epsilon}$ is set to 0.01 unit.

\subsubsection{Query Efficiency $T_{q}$}

According to the results shown in Fig. 14, the Nested-Loop method is the slowest among all. It elaborates all the possible pairs of objects for turning points (but most of them do not contribute to validity intervals). Next, Sample comes the second slowest. We analyze it in Section 6.3.2.

The other three methods have significant improvement over Sample and Nested-Loop. One reason is because of the effectiveness of the pruning techniques, as shown in Fig. 15. For all the real datasets, the pruning ratio are as high as $98.8 \%$. TP-S is less efficient, because some candidates shared by different line segments in trajectory will be fetched multiple times. This drawback is overcome by TP-TS and TP-TS ${ }^{e}$. Notice that gap would be bigger if the query trajectory consists of many tiny line segments. Also, the combined traversal over R-tree in TP-TS and TP-TS save plenty of extra I/O cost, compared to TP-S, shown in Fig. 16.

To get a clearer picture about the efficiency, we measure the time costs for Filtering and Refinement in Fig. 17. TP-TS and TP-TS ${ }^{e}$ are faster than TP-S in both phases. In Filtering,

6. The sampling rate is reasonably high regarding to the trajectory's default length. More details about sampling rates are discussed in Section 6.3.2.

7. http://www.gnu.org/software/gsl/ 


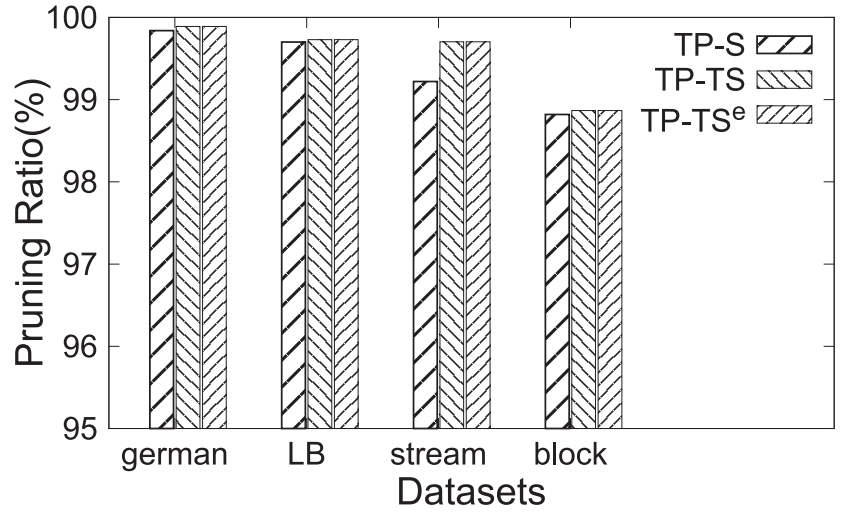

Fig. 15. Pruning ratio vs. datasets.

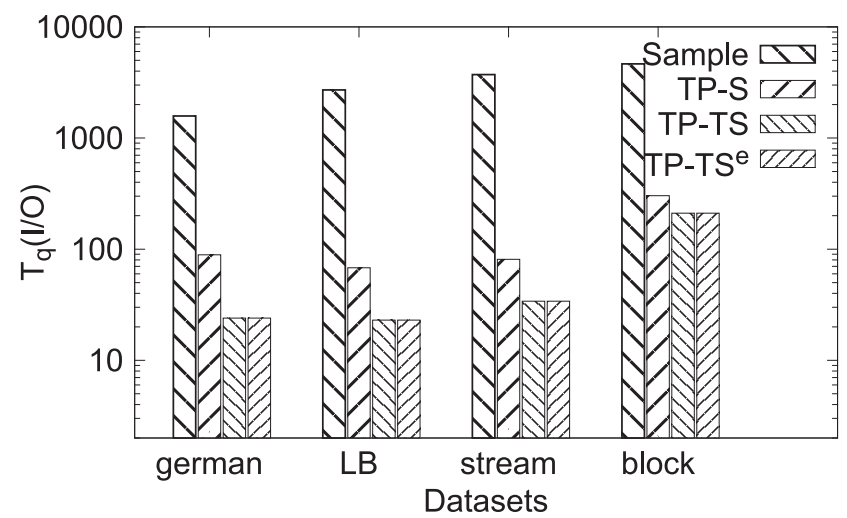

Fig. 16. $T_{q}$ (\# of node access) vs. datasets.

the combined R-tree traversal in TP-TS and TP-TS ${ }^{e}$ save plenty of extra node access, compared to TP-S. The number of node access is shown in Fig. 16. In Refinement, TP-TS and TP-TS ${ }^{e}$ are faster, since they has fewer candidates to handle. The observation is consistent with the fact that TP-TS has a higher pruning ratio, shown in Fig. 15. TP-TSe directly derives turning points by analytical solution, which is more efficient than TP-TS.

\subsubsection{TP-TS vs. Sample}

Efficiency. We test the query efficiency by varying the query length in Fig. 18. The Sample method is slower than others at least one order of magnitude. The costs of others increase stably w.r.t. the query length. The reason can be explained

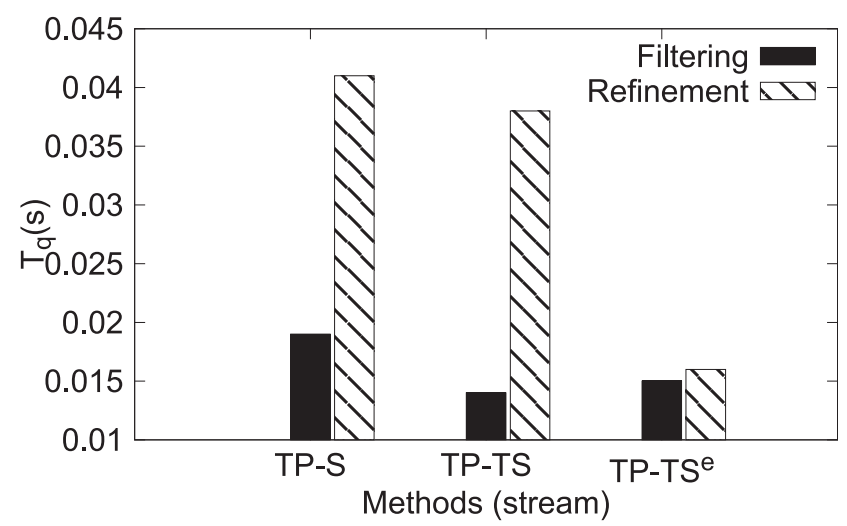

Fig. 17. $T_{q}$ 's breakdown.

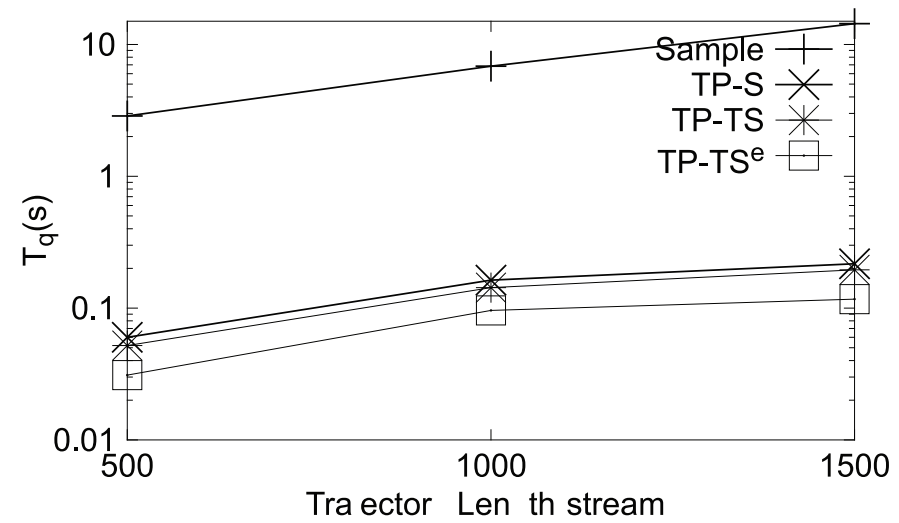

Fig. 18. $T_{q}$ vs. query length.

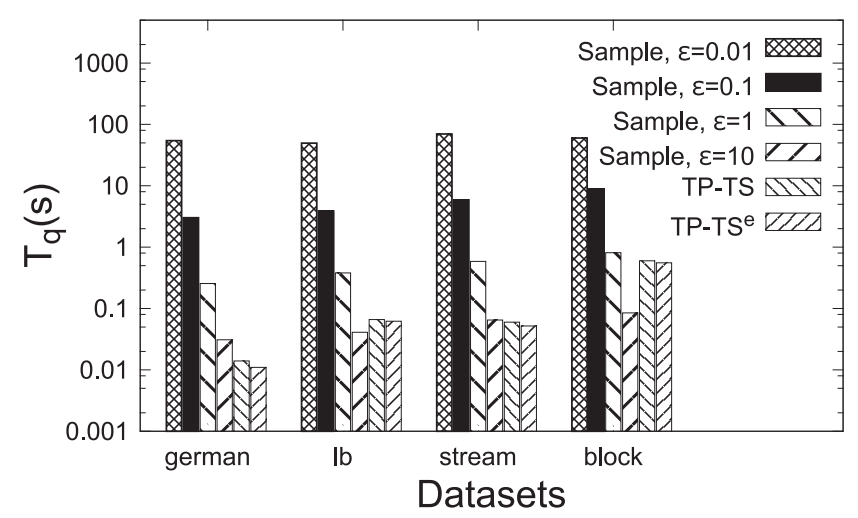

Fig. 19. TP-TS vs. sample $\left(T_{q}\right)$.

in Fig. 16, where Sample incurs much more node access than our methods. We also test the efficiency by varying the sampling interval $\epsilon$ from 0.01 to 10 in Fig. 19. TP-TS outperforms Sample in most of the cases. Sample is faster only when $\epsilon$ is very large (e.g. equal to 10 units). Is it good if large $\epsilon$ is used? The answer is NO. In Table 5, when "Sample, $\epsilon=10$, block", the error score of Sample is as high as 0.443 ! Next, we discuss more detail on the query quality.

Effectiveness. We demonstrate the qualities of Sample and TP-TS in Table 5. TP-TS achieves better query quality than Sample. For Sample, one could choose a very large sampling rate (or a small $\epsilon$ equivalently) for higher quality. But the accompanied burden in efficiency also increases significantly. For example, Sample's query time (when $\epsilon=0.01$ ) is already 100 times slower than that of TP-TS.

We also test the error score of simplifying the imprecise regions by precise points, as mentioned in the introduction. For germany dataset, the error is as high as 0.76 ! In applications such as safety sailing, the simplified solution could be harmful.

TABLE 5

TP-TS vs. Sample (Error)

\begin{tabular}{|c|c|c|c|c|c|}
\hline \multirow{2}{*}{ Datasets } & \multicolumn{4}{|c|}{ Sample } & \multirow{2}{*}{ TP-TS } \\
\cline { 2 - 5 } & $\epsilon=0.01$ & $\epsilon=0.1$ & $\epsilon=1$ & $\epsilon=10$ & \\
\hline german & 0.00340 & 0.00457 & 0.01528 & 0.12310 & $\mathbf{6 . 6 2 e - 6}$ \\
\hline LB & 0.00005 & 0.00029 & 0.00257 & 0.02672 & $\mathbf{5 . 9 0 e - 5}$ \\
\hline stream & 0.00059 & 0.00090 & 0.00298 & 0.03962 & $\mathbf{6 . 0 6 e - 4}$ \\
\hline block & 0.01872 & 0.02541 & 0.08516 & 0.44310 & $\mathbf{5 . 8 0 e - 4}$ \\
\hline
\end{tabular}




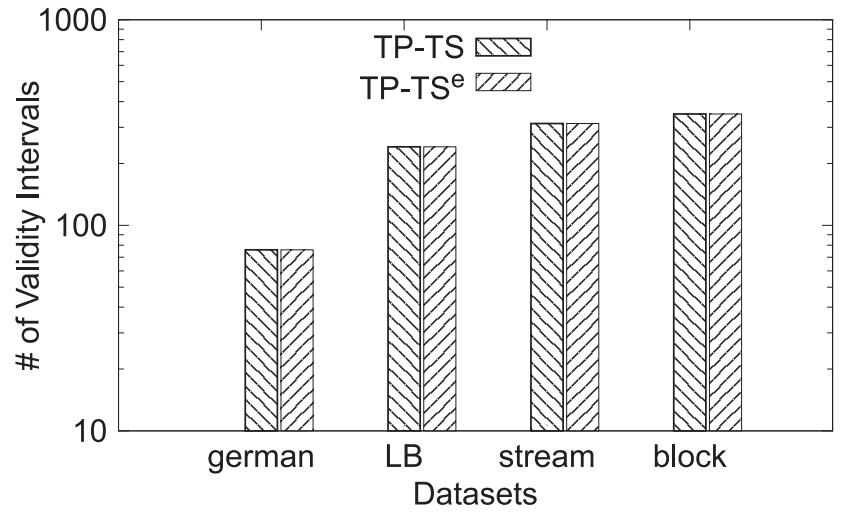

Fig. 20. $\sharp$ of validity intervals vs. datasets (TP-TS).

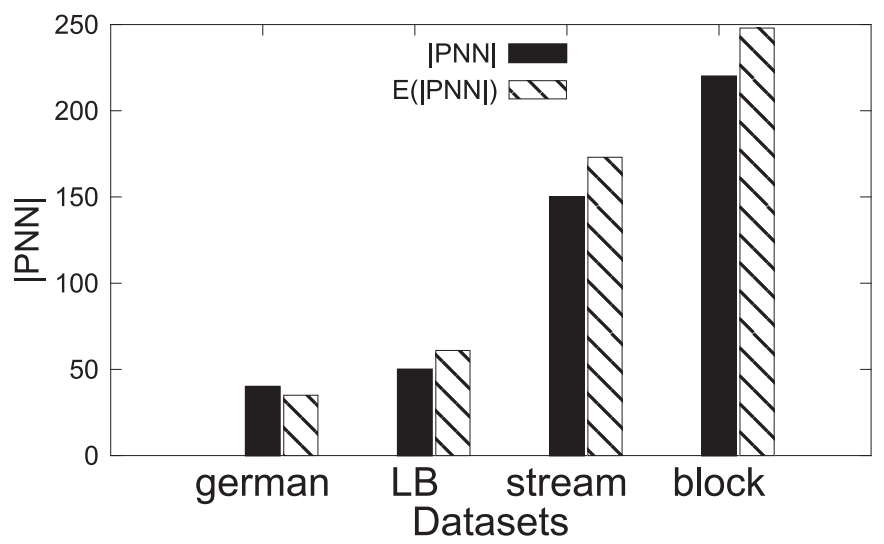

Fig. 21. Estimation of $|P N N|$.

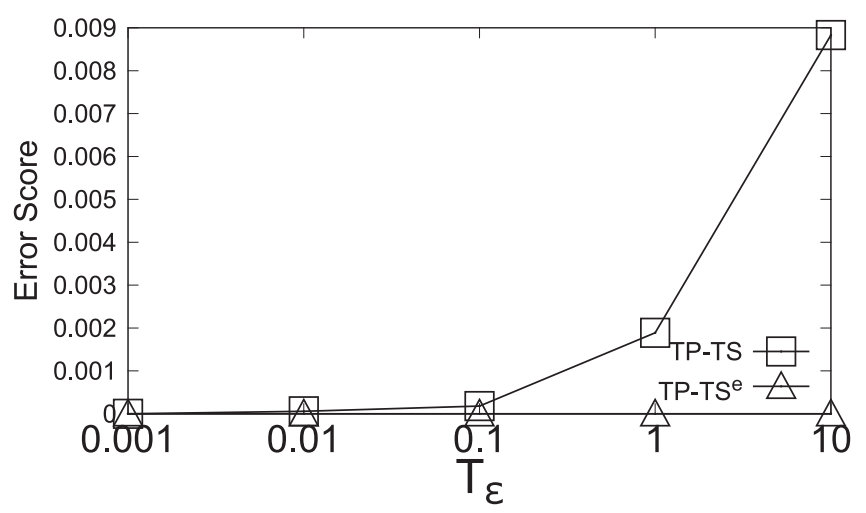

Fig. 22. Error vs. precision (stream).

\subsubsection{Analysis of TPNN}

Observed from Fig. 20, the number of validity intervals increases with the size of the datasets. TP-TS ${ }^{e}$ has the same number of validity intervals, which means the approximate calculation is capable of deriving the turning points within a limited precision $T_{\epsilon}$.

We also test our proposed analysis model in Fig. 21. We split the domain into $25 \times 25$ squares and use average parameters as input. The number of PNNs increases with the size of datasets. In all tested cases, the error rate is within $5 \%$, which shows a high accuracy of the selectivity estimation.

We test the error score of the TP-TS w.r.t. the increase of precision $T_{\epsilon}$. As shown in Fig. 22, when $T_{\epsilon}<0.1$, the error score of TP-TS is quite close to the value of TP-TSe,

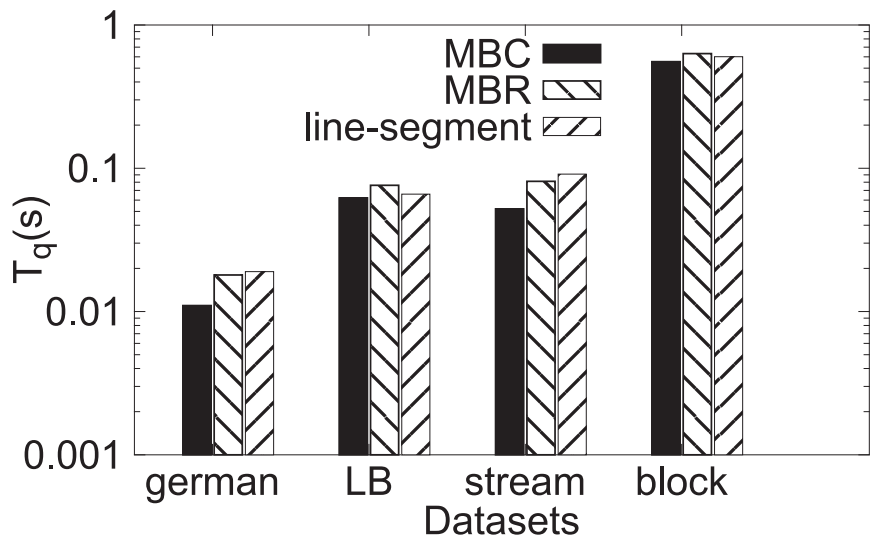

Fig. 23. $T_{q}$ vs. shapes (TP-TS).

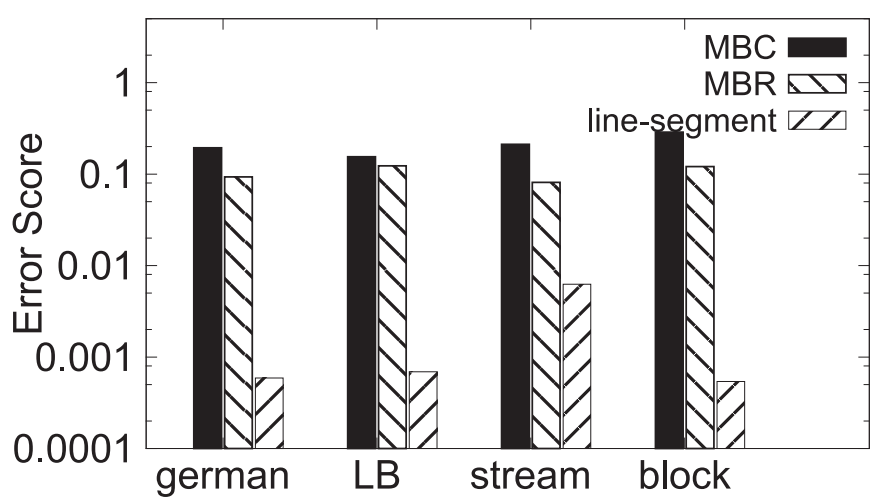

Fig. 24. Error vs. shapes (TP-TS).

which is 0 . This offers us flexibility in choosing the parameter $T_{\epsilon}$. When $T_{\epsilon}>0.1$, the error score increases significantly w.r.t. $T_{\epsilon}$. In our implementation, we set $T_{\epsilon}$ to 0.01 . It is possible to sacrifice some precision for a faster query execution. However, the quality will decrease accordingly. More details are omitted due to page limit.

\subsubsection{Objects with Different Shaped Imprecise Regions}

We model the moving objects on a road network by an imprecise region, whose shape is a line segment. For experiments, we reuse the 4 real rectangular datasets by using each rectangle's two opposite corners as two end-points of a line segment. Then, we test how the quality will be affected by representing the line segment with its enclosed $\mathrm{MBC}$ or $M B R$ (Minimum Bounding Rectangle). We also investigate how the query performance varies for the three different shapes: circle, rectangle, and line segment.

The queries are implemented by TP-TS method. Fig. 23 shows that the $T_{q} \mathrm{~S}$ are similar for the three shapes we have tested. $T_{q}$ on the circular dataset is a little bit faster, as the $\max / \mathrm{min}$ distance evaluation for circular objects requires less distance comparisons than the other two.

However, to approximate a line segment by its MBC or MBR would decrease the query quality. In Fig. 24, the approximated $\mathrm{MBC}^{\prime}$ error is as high as 0.15 . The error of MBR is lower than MBC, because a rectangle has smaller dead space than a circle while enclosing a line segment.

Compared to the result over line segments, the MBC or MBR method's quality is low. In real deployment, if the object could be well represented by its MBC or MBR, we 
suggest to use MBC or MBR for better efficiency. Otherwise, the shape of objects should be considered to achieve better query quality.

In summary, we have shown that TP-TS, as well as its variant TP-TSe, are both efficient and effective. TP-TS is also capable of supporting TPNNQ over arbitrary shaped imprecise regions.

\section{Related Work}

In this section, we review the related work on moving nearest neighbor queries (Section 7.1), as well as the evaluation of trajectory nearest neighbor queries for imprecise location data (Section 7.2).

\subsection{Moving Nearest Neighbor Query}

Nearest neighbor (NN) query for moving query points is a well studied topic [26], [27], [28], [15]. Most existing works focus on reducing the computational cost at the server. They fall into two major categories.

The first category does not require the user's entire trajectory in advance [26], [27], [28], but processes the query online (multiple times) based on the user's moving location.

Song and Roussopoulos [26] propose sampling techniques to answer the moving NN query. They study how to calculate the upper-bound distance within which the moving point does not issue a new query to the server. Some others [27], [28] use validity region and validity time for the query answer of moving points. Voronoi cells are used to represent the validity region. The query answer becomes invalid if the validity time is expired or the user leaves the validity region.

Other types of nearest neighbor queries, like group nearest neighbor query [29], continuous nearest neighbor query [30], expected nearest neighbor query [31] have also been proposed. In these works, the query input is limited to precise points.

The second category assumes that the user's trajectory is known in advance. It evaluates the query only once [15]. In particular, the route of the query point is split into sub-line-segments, such that the $N N$ answer within the same sub-line-segment remains unchanged. A perpendicular bisector $\perp\left(p_{i}, p_{j}\right)$ between two points $p_{i}$ and $p_{j}$ is used to partition the trajectory query into two sub-trajectories, one being definitely closer to $p_{i}$ and the other being definitely closer to $p_{j}$.

The query trajectory in our TPNNQ setting, such as a flight route or a pipeline, is known in advance. However, the exising technique [15] is not applicable to our problem on imprecise location data. As shown in Fig. 2, some segments like $\left[s_{1}, s_{2}\right]$ can have multiple PNNs and it is challenging to derive them.

The bisector for imprecise objects has been addressed by a few works recently. They use bisectors for specific shapes (circles [9], [10], rectangles [11]) to determine the dominance relationship between objects. This paper distinguishes itself from these works in several important aspects.

First, the query studied in this paper is issued for a trajectory, but not for a single object. Second, the $u$-bisector defined in this paper is extended to support arbitrary shaped imprecise objects. It is however unknown how the existing bisectors [9]-[11] can be generalized for similar purposes. Third, our query evaluation partitions the query trajectory into several segments each of which has its own answer set. In contrast, these previous works [9]-[11] do not partition their query objects.

\subsection{Trajectory Nearest Neighbor Query over Uncertain Data}

Only a few works have addressed trajectory queries over imprecise data. Chen et al. [12] study the problem of updating answers for continuous probabilistic nearest neighbor queries in the server was studied. Computational overhead is saved if the query answers are within specific probabilistic bounds. Zheng et al. [32] study range queries over trajectory data. Trajcevski et al. [13] investigate the problem of efficiently executing continuous $N N$ queries for uncertain moving objects trajectories. Zheng et al. [14] study two variants of k-NN query for fuzzy objects. They return the qualified objects satisfying a probabilistic distance threshold or a range of probability thresholds, respectively.

We use the imprecise region model in this paper. It allows us to know which object may be the closest to a given trajectory. In contrast, the uncertainty model described in [12]-[14] contains a probability distribution, which describes the chance that an imprecise object is located in each point in the imprecise region. With this more complex uncertainty model, it is possible to quantify the probability that an imprecise object is the nearest neighbor of any point in a given trajectory. Note such a problem is beyond the scope of this paper and therefore we leave it for future research.

Park et al. [8] study a similar problem as we do in this paper. They also use an imprecise region to model the locations of an object and compute the object closest to a given query segment. However, they only compute and return the definite nearest neighbors but ignore objects that may be the closest. This simplification renders significant answer loss in the query result. Also, unlike our solution in this paper, the techniques in [8] are specific to circular objects and are inapplicable to arbitrary shaped imprecise objects.

\section{Conclusion}

In this paper, we study the problem of trajectory possible nearest neighbor query (TPNNQ) over imprecise data. To overcome the low quality and inefficiency in simplified methods, we study the geometric properties of $u$-bisector. Based on that, we design an efficient query evaluation approach that follows the filter-refinement paradigm. We also generalize our solution to arbitrary shaped imprecise data. Further, we propose theoretic analysis to estimate the $T P N N Q$ query result size. We conduct extensive experiments to evaluate our proposals. The results show that our query evaluation approach is efficient and scalable. Meanwhile, our TPNNQ query result size estimation gives very good hints.

In future, we would like to extend our solution to other distance metrics (e.g., Manhattan distance) and support other query variants (e.g., k possible nearest neighbor). We are also interested in generalizing $u$-bisector to find nearest neighbors with probabilistic guarantees. 


\section{ACKNOWLEDGMENTS}

R. Cheng and X. Xie were supported in part by the Research Grants Council of Hong Kong (GRF Projects 711309E, 711110) and in part by the University of Hong Kong (Seed Funding Project 201211159083). M. L. Yiu was supported by grant PolyU 5302/12E from Hong Kong RGC. The authors would like to thank the anonymous reviewers for their insightful comments.

\section{APPENDIX}

Lemma 8. Given two imprecise objects $O_{i}, O_{j}$ and a linesegment $\mathcal{L}(s, e), O_{j}$ can not be $p \in \mathcal{L}^{\prime}$ s PNN if $O_{j}$ does not overlap with $\odot\left(s, O_{i}\right) \cup \odot\left(e, O_{i}\right)$.

Lemma 9 (Imprecise Region Decomposition.) Given imprecise objects $O_{i}$ and $O_{j}$, if their imprecise regions are decomposed into two sets of sub-regions $P$ and $Q$, say $u_{i}=\left\{u_{i(p)}\right\}_{p \in P}$ and $u_{j}=\left\{u_{j(q)}\right\}_{q \in Q}$, $H_{i}(j)=\cap_{p \in P \wedge q \in Q} H_{i(p)}(j(q))$.

Lemma 10. Given two triangle $\triangle A B C$ and $\triangle A^{\prime \prime} B^{\prime \prime} C^{\prime \prime}, D$ and $D^{\prime \prime}$ are two midpoints on $B C$ and $B^{\prime \prime} C^{\prime \prime}$, respectively. If $|B C|=$ $\left|B^{\prime \prime} C^{\prime \prime}\right|,|A B| \leq\left|A^{\prime \prime} B^{\prime \prime}\right|$ and $|A C| \leq\left|A^{\prime \prime} C^{\prime \prime}\right|$, then $|A D| \leq$ $\left|A^{\prime \prime} D^{\prime \prime}\right|$.

Lemma 11. Given two imprecise objects $O_{i}$ and $O_{j}$, whose imprecise regions are circles: $O_{i}\left(C_{i}, r_{i}\right)$ and $O_{j}\left(C_{j}, r_{j}\right)$. A linesegment $\mathcal{L}(s, e)$ has at most two intersection points with the $u$-bisector: $b_{i}(j)$ and $b_{j}(i)$.

Due to space limit, we put the proofs of $8,9,10$, and 11 in a technical report [19].

\section{REFERENCES}

[1] D. C. Scott. Available Now: A Volcanic Ash Detector for Aircraft [Online]. Available:

http:/ /www.csmonitor.com/World/Europe/2010/0420/Available -now-a-volcanic-ash-detector-for-aircraft

[2] U. S. C. Guard. Announcement of 2011 International Ice Patrol Services [Online]. Available: http://www.uscg.mil/lantarea/iip/docs/AOS $2011 . p d f$

[3] Wikipedia. Active Phased Array Radar [Online]. Available: http:/ / en.wikipedia.org/wiki/Activephased rrayRadar $_{\text {a }}$

[4] Dow Employs GPS for Pipeline Worker Safety [Online]. Available: http:/ / www.automationworld.com/ feature-8577

[5] L. Jesse, R. Janet, G. Edward, and V. Lee, "Effects of habitat on GPS collar performance: Using data screening to reduce location error," J. Appl. Ecol., vol. 44, no. 3, pp. 663-671, Jun. 2007.

[6] R. Cheng, D. V. Kalashnikov, and S. Prabhakar, "Querying imprecise data in moving object environments," IEEE Trans. Knowl. Data Eng., vol. 16, no. 9, pp. 1112-1127, Sept. 2004.

[7] H. Lu1, B. Yang, and C. S. Jensen, "Spatio-temporal joins on symbolic indoor tracking data," in Proc. IEEE 27th ICDE, Hannover, Germany, 2011.

[8] K. Park, H. Choo, and P. Valduriez, "A scalable energy-efficient continuous nearest neighbor search in wireless broadcast systems," Wireless Netw., vol. 16, no. 4, pp. 1011-1031, 2010.

[9] R. Cheng, X. Xie, M. L. Yiu, J. Chen, and L. Sun, “UV-diagram: A Voronoi diagram for uncertain data," in Proc. ICDE, Long Beach, CA, USA, 2010.

[10] X. Lian and L. Chen, "Efficient processing of probabilistic reverse nearest neighbor queries over uncertain data," VLDB J, vol. 18, no. 3, pp. 787-808, 2009.

[11] M. A. Cheema, X. Lin, W. Wang, W. Zhang, and J. Pei, "Probabilistic reverse nearest neighbor queries on uncertain data," IEEE Trans. Knowl. Data Eng., vol. 22, no. 4, pp. 550-564, Apr. 2010.
[12] J. Chen, R. Cheng, M. Mokbel, and C. Chow, "Scalable processing of snapshot and continuous nearest-neighbor queries over one-dimensional uncertain data," $V L D B$ J., vol. 18, no. 5, pp. 1219-1240, 2009.

[13] G. Trajcevski, R. Tamassia, H. Ding, P. Scheuermann, and I. F. Cruz, "Continuous probabilistic nearest-neighbor queries for uncertain trajectories," in Proc. 12th Int. Conf. EDBT, Saint Petersburg, Russia, 2009, pp. 874-885.

[14] K. Zheng, G. P. C. Fung, and X. Zhou, "K-nearest neighbor search for fuzzy objects," in Proc. ACM SIGMOD, Indianapolis, IN, USA, 2010

[15] Y. Tao, D. Papadias, and Q. Shen, "Continuous nearest neighbor search," in Proc. 28th Int. Conf. VLDB, Hong Kong, China, 2002.

[16] X. Xie, R. Cheng, and M. L. Yiu, "Evaluating trajectory queries over imprecise location data," in Proc. 24th Int. Conf. SSDBM, Chania, Greece, 2012.

[17] M. Kolahdouzan and C. Shahabi, "Voronoi-based k nearest neighbor search for spatial network databases," in Proc. 30th Int. Conf. $V L D B$, Toronto, ON, Canada, 2004.

[18] H. Samet, Foundations of Multidimensional and Metric Data Structures. San Francisco, CA, USA: Morgan Kaufmann, Inc., 2005.

[19] X. Xie, M. L. Yiu, R. Cheng, and H. Lu. (2012). "Trajectory Possible Nearest Neighbor Queries Over Imprecise Location Data," Tech. Rep. [Online]. Available: http://dbtr.cs.aau.dk/DBPublications/DBTR-33.pdf

[20] K. Nikodem, "Midpoint convex functions majorized by midpoint concave functions," Aequationes Math., vol. 32, no. 1, pp. 45-51, 1987.

[21] A. Okabe, B. Boots, K. Sugihara, and S. Chiu, Spatial Tessellations: Concepts and Applications of Voronoi Diagrams, 2nd ed. New York, NY, USA: Wiley, 2000.

[22] W. Stallings, Wireless Communications \& Networks, 2nd ed. Upper Saddle River, NJ, USA: Prentice-Hall, Inc., 2004.

[23] X. Xie, R. Cheng, M. Yiu, L. Sun, and J. Chen, "UV-diagram: A Voronoi diagram for uncertain spatial databases," VLDB J., vol. 22, no. 3, pp. 319-344, 2013.

[24] M. Hadjieleftheriou. Spatial Index Library Version 0.44.2b [Online]. Available: http://u-foria.org/marioh/spatialindex/index.html

[25] P.-N. Tan, M. Steinbach, and V. Kumar, Introduction to Data Mining. Boston, MA, USA: Addison-Wesley, 2006.

[26] Z. Song and N. Roussopoulos, "K-nearest neighbor search for moving query point," in Proc. 7th Int. SSTD, Redondo Beach, CA, USA, 2001

[27] Z. Baihua and L. Dik, "Semantic caching in location-dependent query processing," in Proc. Adv. SSTD, Berlin, Germany: Springer, 2001.

[28] J. Zhang, M. Zhu, D. Papadias, Y. Tao, and D. L. Lee, "Locationbased spatial queries," in Proc. ACM SIGMOD, San Diego, CA, USA, 2003

[29] X. Lian and L. Chen, "Probabilistic group nearest neighbor queries in uncertain databases," IEEE Trans. Knowl. Data Eng., vol. 20, no. 6, pp. 809-824, Jun. 2008.

[30] Y. Jin, R. Cheng, B. Kao, K.-Y. Lam, and Y. Zhang, "A filter-based protocol for continuous queries over imprecise location data," in Proc. 21st ACM Int. CIKM, New York, NY, USA, 2012.

[31] P. K. Agarwal, A. Efrat, S. Sankararaman, and W. Zhang, "Nearest-neighbor searching under uncertainty," in Proc. 31st Symp. PODS, Scottsdale, AZ, USA, 2012.

[32] K. Zheng, G. Trajcevski, X. Zhou, and P. Scheuermann, "Probabilistic range queries for uncertain trajectories on road networks," in Proc. 14th Int. Conf. EDBT, Uppsala, Sweden, 2011.

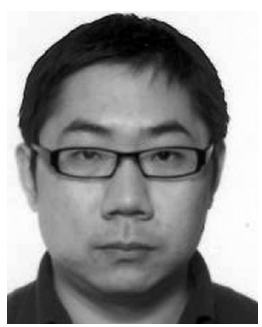

Xike Xie received the B.Sc. and M.Sc. degrees from the Xi'an Jiaotong University, China, in 2003 and 2006, respectively, and the Ph.D. degree in computer science from the University of Hong Kong, Hong Kong, in 2012. He is currently a Research Assistant Professor in the Department of Computer Science, Aalborg University, Denmark. His current research interests include data uncertainty, spatiotemporal databases, and mobile computing. He is a member of the IEEE and ACM. 


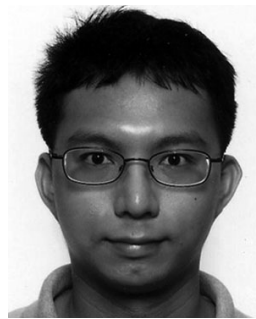

Man L. Yiu received the bachelor's degree in computer engineering and the Ph.D. degree in computer science from the University of Hong Kong, Hong Kong, in 2002 and 2006, respectively. Prior to his current post, he was with the Aalborg University, Denmark, for three years from 2006. He is now an Assistant Professor in the Department of Computing, Hong Kong Polytechnic University, Hong Kong. His current research interests include management of complex data, in particular query processing topics on spatiotemporal data and multidimensional data.

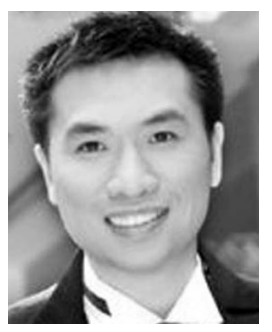

Reynold Cheng received the B.E. degree in computer engineering and the M.Phil. degree in computer science and information systems from the University of Hong Kong (HKU), Hong Kong in 1998 and 2000, respectively, and the M.Sc. and Ph.D. degrees from the Department of Computer Science, Purdue University, West Lafayette, IN, USA, in 2003 and 2005, respectively. $\mathrm{He}$ is an Associate Professor in the Department of Computer Science, HKU. He was the recipient of the 2010 Research Output Prize from the Department of Computer Science of HKU. From 2005 to 2008, he was an Assistant Professor in the Department of Computing, Hong Kong Polytechnic University, Hong Kong, where he received two Performance Awards. He is a member of the IEEE, ACM, ACM SIGMOD, and UPE. He has served on the program committees and review panels for leading database conferences and journals. $\mathrm{He}$ is a member of the Editorial Board of Information Systems and DAPD journal. $\mathrm{He}$ is also a Guest Editor for a special issue in TKDE. His current research interests include database management, as well as querying and mining of uncertain data.

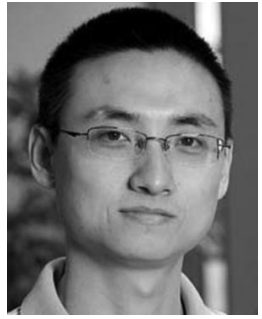

Hua Lu received the B.Sc. and M.Sc. degrees from the Peking University, China, in 1998 and 2001, respectively, and the Ph.D. degree in computer science from the National University of Singapore, Singapore, in 2007. Currently, he is an Associate Professor in the Department of Computer Science, Aalborg University, Denmark. His current research interests include databases, geographic information systems, as well as mobile computing. Recently, he is with indoor spatial awareness, complex queries on spatial data with heterogeneous attributes, and location privacy in mobile services. He has served on the program committees for conferences and workshops including ICDE, ACM SIGSPATIAL GIS, SSTD, MDM, PAKDD, APWeb, and MobiDE. He is a PC Co-Chair or Vice Chair for ISA 2011, MUE 2011, and MDM 2012. He is a member of the IEEE.

$\triangleright$ For more information on this or any other computing topic, please visit our Digital Library at www.computer.org/publications/dlib. 